\title{
Triggering antitumoural drug release and gene expression by magnetic hyperthermia
}

\author{
María Moros a,b,1 , Javier Idiago-López ${ }^{\text {a,b,1 }}$, Laura Asín ${ }^{\text {a,b }}$, Eduardo Moreno-Antolín a , Lilianne Beola ${ }^{\text {a }}$, \\ Valeria Grazú $^{\text {a,b }}$, Raluca M. Fratila ${ }^{\text {a,b,* }}$, Lucía Gutiérrez ${ }^{\text {b,c,* }}$, Jesús Martínez de la Fuente ${ }^{\text {a,b }}$ \\ a Instituto de Ciencia de Materiales de Aragón (ICMA), CSIC/Universidad de Zaragoza, Zaragoza, Spain \\ ${ }^{\mathrm{b}}$ Centro de Investigación Biomédica en Red de Bioingeniería, Biomateriales y Nanomedicina (CIBER-BBN), Spain \\ c Instituto Universitario de Nanociencia de Aragón (INA), Universidad de Zaragoza, Zaragoza, Spain
}

\section{A R T I C L E I N F O}

\section{Article history:}

Received 14 May 2018

Received in revised form 6 September 2018

Accepted 8 October 2018

Available online $\mathrm{xxxx}$

\section{Keywords:}

Magnetic nanoparticles

Liposomes

Gene therapy

Switchable gates

Hydrogel

Polymer

Cancer

\begin{abstract}
A B S T R A C T
Magnetic nanoparticles (MNPs) are promising tools for a wide array of biomedical applications. One of their most outstanding properties is the ability to generate heat when exposed to alternating magnetic fields, usually exploited in magnetic hyperthermia therapy of cancer. In this contribution, we provide a critical review of the use of MNPs and magnetic hyperthermia as drug release and gene expression triggers for cancer therapy. Several strategies for the release of chemotherapeutic drugs from thermo-responsive matrices are discussed, providing representative examples of their application at different levels (from proof of concept to in vivo applications). The potential of magnetic hyperthermia to promote in situ expression of therapeutic genes using vectors that contain heat-responsive promoters is also reviewed in the context of cancer gene therapy.
\end{abstract}

(c) 2018 Published by Elsevier B.V.

\section{Contents}

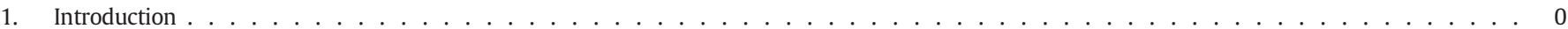

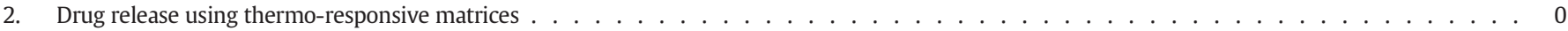

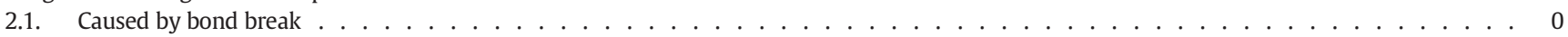

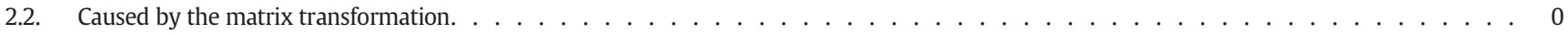

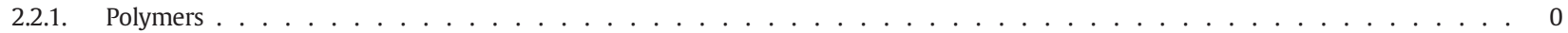

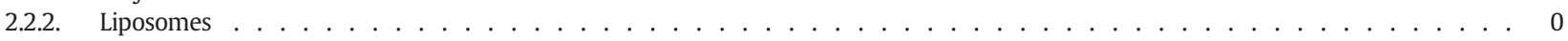

2.3. Using switchable gates . . . . . . . . . . . . . . . . . . . . . . . . . . . . . . . . . . . . . 0

2.4. Caused by matrix degradation $\ldots \ldots \ldots \ldots \ldots$

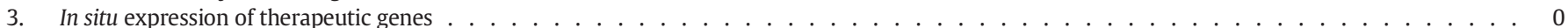

Abbreviations: 5-FC, 5-fluorocytosine; 5-FU, 5-fluorouracil; AD-MSCs, Adipose-derived mesenchymal stem cells; AMF, Alternating magnetic field; DA, Diels-Alder; DDMH, Dual-drug-

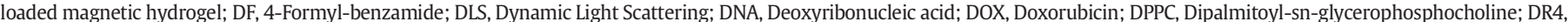

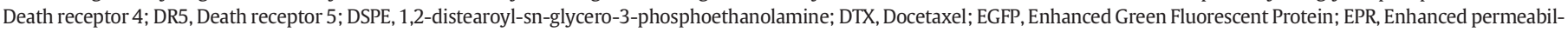

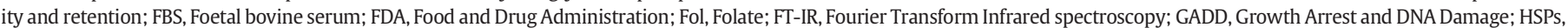

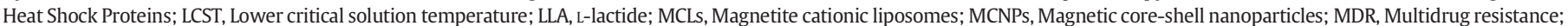

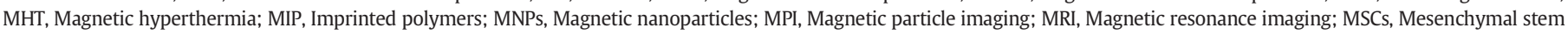

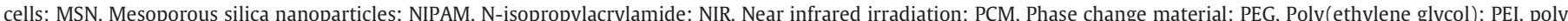

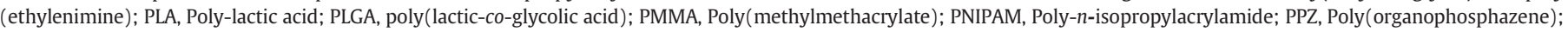

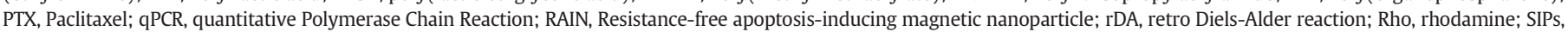
Self-immolative polymers; TEM, Transmission Electron Microscopy; Tm, Melting temperature; TNF, Tumour necrosis factor; TRAIL, TNF-related apoptosis-inducing ligand.

* Corresponding author at: Instituto de Ciencia de Materiales de Aragón (ICMA), Campus Rio Ebro, Edificio I+D, C/Mariano Esquillor s/n, 50018, Zaragoza, Spain

E-mail addresses: rfratila@unizar.es (R.M. Fratila), lu@unizar.es (L. Gutiérrez).

1 Equal contribution. 
4. Conclusions and future remarks

Acknowledgements

References.

\section{Introduction}

Magnetic nanoparticles (MNPs) emerged more than two decades ago as promising tools for a wide array of biomedical applications [1]. Several magnetic materials have been studied although the most popular ones are iron oxides, due to their excellent biocompatibility properties. These materials have been approved by the Food and Drug Administration (FDA) of the USA as magnetic resonance imaging (MRI) contrast agents [2] and are being extensively investigated as drug delivery agents using external magnetic fields [3] and as heat mediators for cancer treatment using alternating magnetic fields (AMF) [4]. This last biomedical application is usually described as magnetic hyperthermia (MHT).

Magnetic hyperthermia is a promising therapy for the localized treatment of cancer. Given that cancer cells are more sensitive to heat than normal cells, MNPs are introduced in the tumour area and used as heating agents to increase the local temperature to induce cell death or alter the growth and differentiation of the cancer cells. The production of energy in the form of heat is based on the reorientation of the magnetic moments of the MNPs under exposure to an AMF [5]. In small monodomain particles, this reorientation can occur by two mechanisms [6]: Néel rotation, when the magnetic moment rotates overcoming the anisotropy energy barrier [7], and Brown rotation, when the whole particle rotates creating frictional losses with the environment [8].

Despite the immense amount of research being performed in this area, cancer treatment by magnetic hyperthermia still faces many challenges until it can be adopted as a widely used approach [9]. Some examples are the difficulties to achieve a uniform thermal profile within the tumour [10], the lack of knowledge of the molecular mechanisms involved in the cellular damage [11], the reticence from the medical community and the difficulties to access the technical facilities needed for the treatment [12].

Recently, new approaches based on the use of magnetic hyperthermia for cancer treatment that go one step further than traditional magnetic hyperthermia have been developed [13]. Instead of focusing just on the heat generation to induce cell death, the main aim is to use the temperature increase as a stimulus able to trigger the release or the activation of therapeutic molecules.

The different stimuli that can be used for triggering on demand release of the drug/molecule of interest at the desired target site can be classified in two major categories: internal and external [14]. Internal stimuli approaches are based on the different physicochemical situations that can be present at the target site. Focusing on tumours, differences in the $\mathrm{pH}[15,16]$ and/or the overexpression of some enzymes $[17,18]$ can be used as internal stimuli to control the release of the content of the nanomaterial acting as carrier. Although this idea seems to be promising there are difficulties for the design of an effective drug release system. The differences in the $\mathrm{pH}$ or the enzyme concentration should be large enough to avoid the premature release of the cargo in the blood stream or in any other not desired environment. This problem could be solved with the use of external stimuli, which can be switched on to achieve the optimal spatio-temporal control of the drug release. In addition to the alternating magnetic fields described above to release energy from magnetic nanoparticles, other non-invasive external stimuli, such as light or ultrasounds, have been successfully used to promote drug release from nanoparticulate systems [19-21]. Absorbed light with wavelengths ranging from the ultraviolet (UV) to the near infrared (NIR) region of the spectrum can lead to the release of the drug from the nanocarrier system by different mechanisms, including photocleavage, photoswitching, photoisomerization, heat generation, or production of singlet oxygen. However, ultraviolet-visible light suffers from low penetration depth, although this aspect can be improved by using near infrared (NIR)-responsive systems. Ultrasound waves can also promote drug delivery from nanocarriers through mechanical and/ or thermal effects. The use of high-intensity focused ultrasounds (HIFU) for the release of chemotherapeutic drugs from thermosensitive carriers (ThermoDox ${ }^{\circledR}$ ) is currently under clinical investigation for the treatment of liver tumours [22]. Nevertheless, the use of high-frequency ultrasounds that might be required to ensure high penetration depths could lead to adverse effects such as thermal damage of the healthy tissue [20].

There are several advantages related to the use of external stimuli for the release or activation of therapeutic agents. First, the therapeutic molecules are protected from premature degradation in the body before reaching the targeted area. In this sense, a high stability (both physical and chemical) of the polymers and liposomes generally used for these approaches is of paramount importance. For this purpose, the composition of these nanostructures plays a key role in avoiding the passive permeation of the encapsulated or bound agents. Another advantage associated to the use of external stimuli to release or activate therapeutic agents is that it also allows the better control of the timing, meaning that they are active at the desired time point.

The use of alternating magnetic fields as external stimuli presents several additional advantages. First, using field gradients from MPI (Magnetic Particle Imaging) equipment it is possible to have a careful control of the spatial localization of the release of therapeutic molecules, which avoids problems related to side effects and systemic cytotoxicity [23]. Besides, synergistic effects between hyperthermia and chemotherapy have been described [24], as the temperature increase is able to make the tumour cells more sensitive to some therapeutic molecules $[25,26]$.

Two main design strategies are being followed in the development of heat-responsive nanostructures to trigger the release of antitumoural agents mediated by magnetic hyperthermia. The first one is based on the link or encapsulation of a drug in a thermo-responsive matrix that will be transformed once the temperature is raised allowing the release of the cargo. The second one relies on the expression of genes, using vectors containing inducible promoters, such as heat-responsive ones.

The main idea behind the use of thermo-responsive matrices is that the heat induced by the MNPs under the AMF is able to transform the matrix in which the therapeutic agent is carried. Four major strategies are being followed to this end (Fig. 1). The first one is the break or destabilization of the bond between the therapeutic molecule and the matrix, allowing its release (Fig. 1A). The second strategy would take advantage of the heat-induced transformation of the thermo-responsive matrix (Fig. 1B), allowing the escape of the therapeutic drug from the matrix, mainly based on the use of thermo-responsive polymers or magnetoliposomes. A possible mechanism for this approach would be related to the use of materials with a conformational transition temperature above the body temperature. The third approach is based on the use of a porous material, where the therapeutic agents are retained, whose pores are blocked by a thermo-responsive material (Fig. 1C). The application of heat induces a transformation on the pore coating allowing the release of the therapeutic agents. This approach is usually referred to as "switchable gates". Finally, a fourth and less common strategy consists in the degradation of the matrix, allowing the release of its cargo, using thermo-sensitive polymers that undergo a dissolution process at higher temperatures (Fig. 1D). 


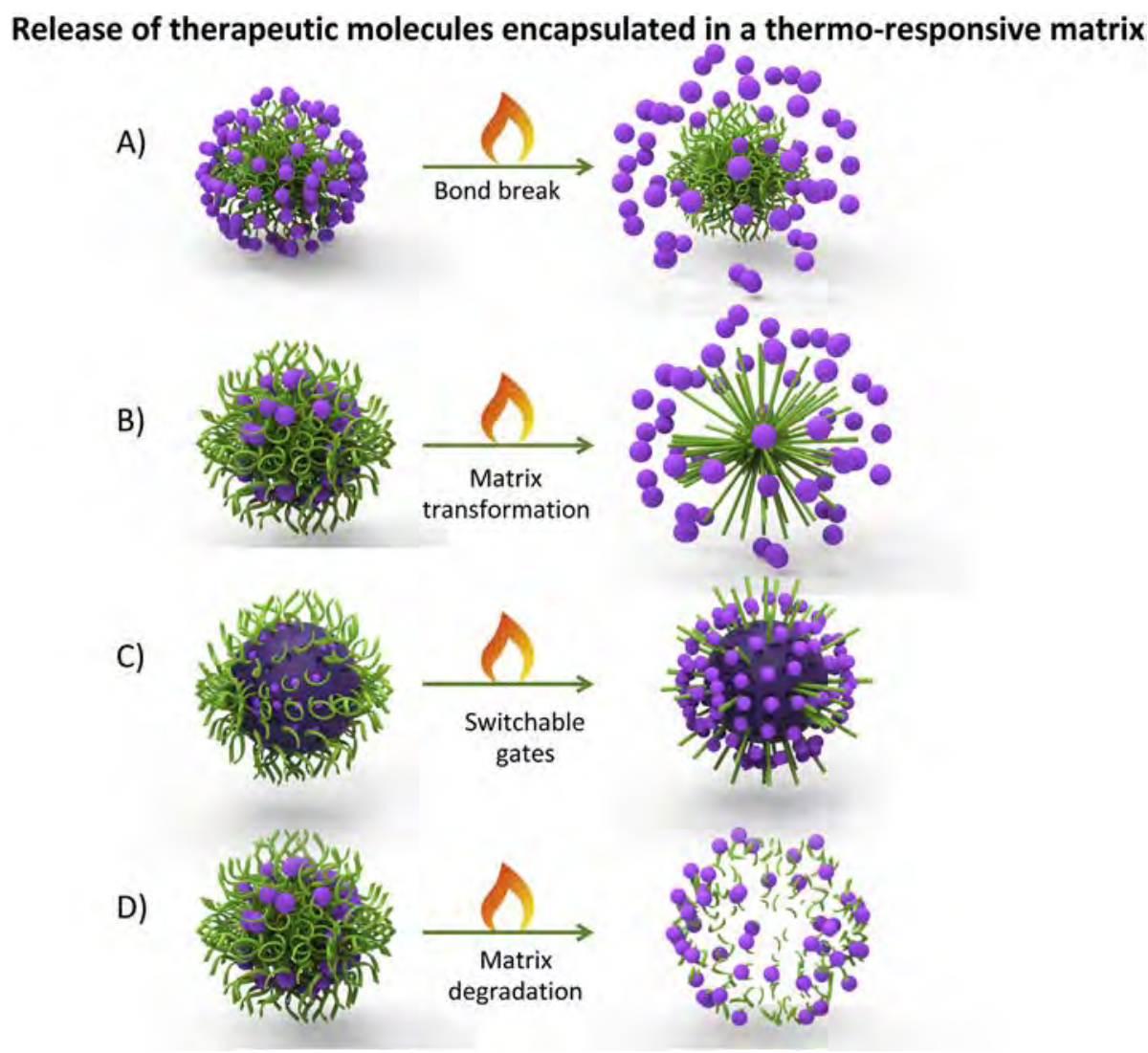

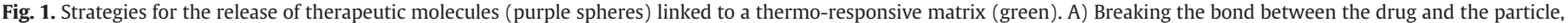
B) Transforming the matrix structure, C) Using the thermo-responsive matrix to open/close the pores from a mesoporous material and D) Degrading the matrix structure.

Instead of carrying a drug to be locally released, an alternative strategy aims at the in situ expression of a therapeutic gene, for example a suicide gene able to cause cellular death. This gene would be expressed when a heat-responsive promoter is activated (Fig. 2). Promoters are carefully chosen to initiate the transcription of the gene only when the increase in temperature occurs resulting in the expression of the therapeutic genes in a controllable way. This elegant approach allows the in situ production of the gene with both spatial and temporal control.

In this manuscript we will review the scientific literature regarding the use of magnetic hyperthermia to trigger the release of drugs or to activate therapeutic molecules for cancer treatment. The presented results are focused only on the use of iron oxide nanoparticles as magnetic mediators for the heat production due to their low toxicity. The results will be classified in two groups: a first group gathering representative examples of release of therapeutic drugs from a thermo-responsive matrix and a second one focused on the activation of gene expression through the increase in temperature. In both approaches, once the most relevant properties of each nanostructure and the key factors involved in the heat-triggered drug release using magnetic hyperthermia are

\section{In situ generation of therapeutic genes}

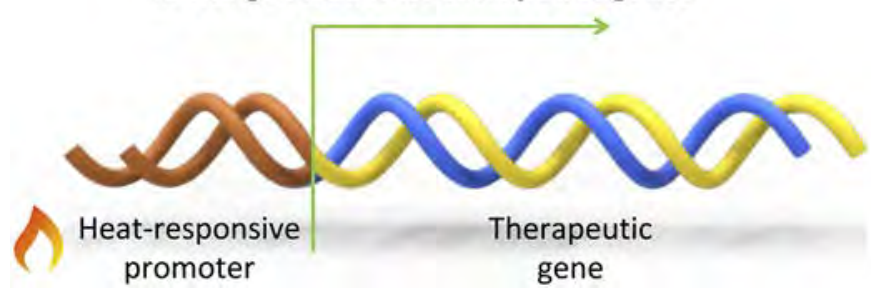

Fig. 2. Schematic representation for the in situ generation of therapeutic genes. A heatresponsive promoter activates the expression of a therapeutic gene. addressed, in vitro and in vivo reports will be discussed, highlighting relevant aspects to consider for future developments.

\section{Drug release using thermo-responsive matrices}

\subsection{Caused by bond break}

During the last years, different types of covalent and non-covalent bonds have been used to precisely release on demand diverse molecules upon AMF exposure (Fig. 3). In this sense, instead of using materials that are sensitive to heat, the molecules to be released are bound to the MNPs via bonds that can be broken or destabilized when the temperature is increased.

The advantage of using non-covalent bonds ( $\pi-\pi$ interactions, and hydrogen bonds not based on nucleic acids...) [27-31] is that the drug does not have to be modified, resulting in overall simpler procedures [32]. On the other hand, it is more challenging in terms of preventing a premature release of the drug. Among non-covalent interactions, hydrogen bonds are the most popular (Fig. 3).

Hydrogen bonds can be used to directly bind the drug to the MNP, as reported by Griffete et al. [31]. In this case, 11-nm MNPs containing acrylic acid monomer were coated with molecularly imprinted polymers (MIP) directly grown on their surface, using doxorubicin (DOX) as a template. When applying an $\operatorname{AMF}\left(335 \mathrm{kHz}, 7.17 \mathrm{kA} / \mathrm{m}\right.$ at $\left.37{ }^{\circ} \mathrm{C}\right)$, the DOX was released in the absence of a global temperature elevation, due to the destabilization of the hydrogen bonds between the drug and the MIP. The cumulative release of the DOX under the AMF (60\%) was significantly higher than the one obtained by incubating the MNPs in an external bath at the same temperature. Further, the importance of introducing the DOX in the imprinting process was demonstrated by comparing the release with the one obtained by physically adsorbing DOX in unprinted polymer MNPs. In this case, in the external bath $95 \%$ of the 


\section{Release of therapeutic molecules by bond break}
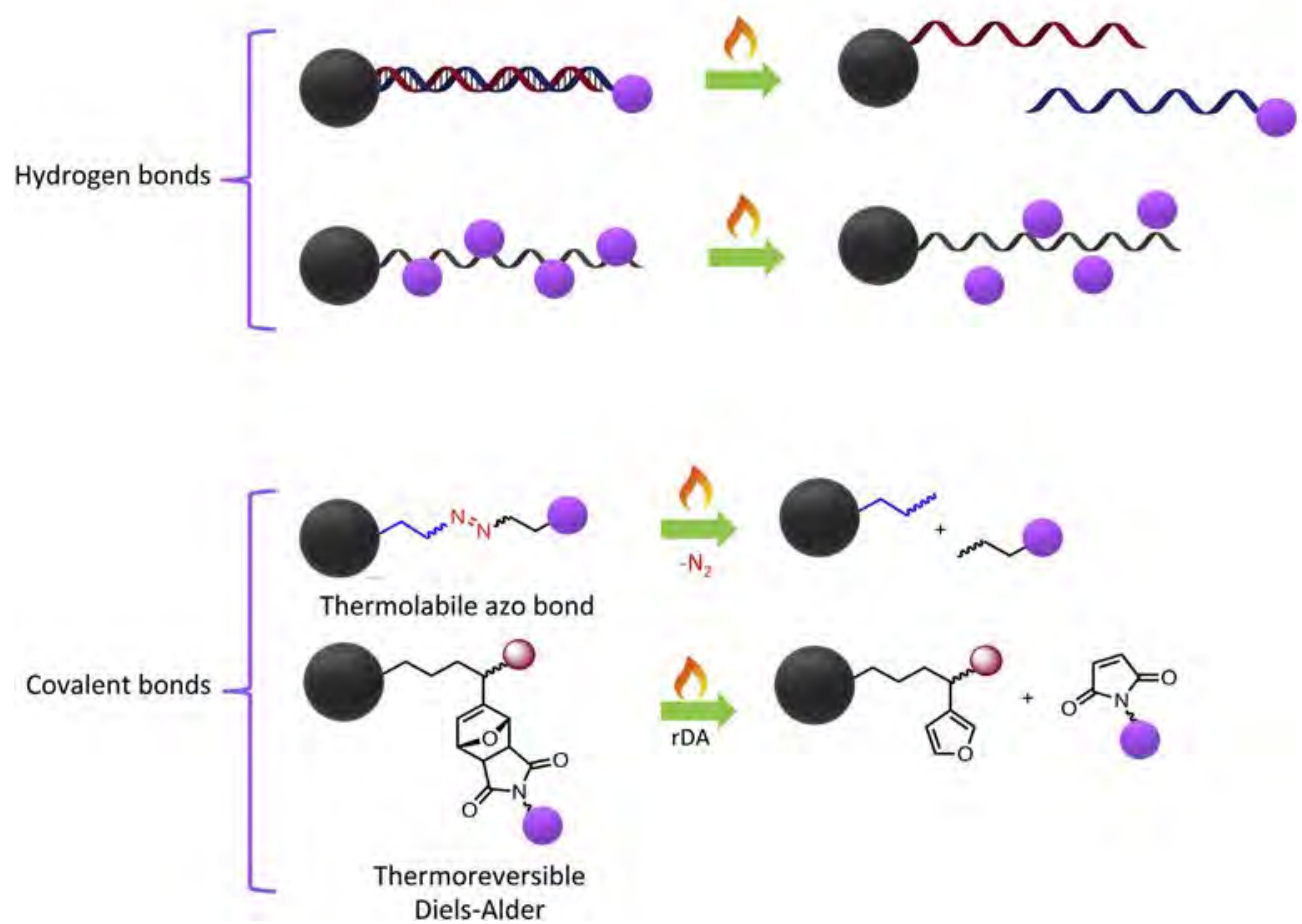

Fig. 3. Schematic representation of drug release strategies based on the break of non-covalent and covalent bonds.

drug was released fast, and the use of an AMF did not offer further advantages. Lastly, the MNPs-MIP caused a $60 \%$ cell viability reduction in PC-3 cells (human prostate cancer cell line) without temperature increase after AMF application $(700 \mathrm{kHz}, 19.95 \mathrm{kA} / \mathrm{m})$; the same viability reduction was shown when using free DOX, without MNPs.

The extremely high specificity of nucleic acids base pairing allows designing complementary DNA (deoxyribonucleic acid) strands that will interact via non-covalent hydrogen-bonding. DNA molecules show high physico-chemical stability, and high rigidity when they stand as a double-strand [33]. Complementary strands can be modified with a drug, which can be released upon local heating caused by AMF application. Depending on the length of the strand and the composition, it is possible to fine-tune the melting temperature ( $\mathrm{Tm})$, that is, the temperature where $50 \%$ of the strands will dehybridize. For instance, Derfus et al. demonstrated the possibility to modulate the DNA release depending of the Tm, by designing MNPs functionalized with a DNA strand; afterwards it was hybridized with complementary DNA strands of different lengths (and consequently diverse $\mathrm{Tm}$ ) bearing fluorophores. Low power electromagnetic field pulses ( $400 \mathrm{kHz}-0.55 \mathrm{~kW}$ ) could trigger the release of the shortest complementary strand, while stronger pulses ( $3 \mathrm{~kW}$ ) were needed to release the longer strand [30]. A similar strategy was used to assess the temperature gradient on the MNP surface upon AMF excitation, by associating it with the DNA denaturation profile [29]. By using three strands of different lengths, each one modified with a diverse fluorophore, it was also possible to correlate these local temperatures with the distance of the DNA from the MNP surface, and consequently to create an accurate spatial mapping of the temperature. Despite the high potential of this triggering strategy, not many examples of it can be found in the literature. Further, many of them are proof of concept reports, where the experiments have been performed in a test tube, but not in vitro or in vivo. [34].

Temperature responsive covalent bonds can be successfully used to release drugs under an AMF in a highly controlled fashion (Fig. 3). To this end, thermo-reversible Diels-Alder (DA) reactions and thermo-labile azo-linkers have been described [13,35-38]. Among the drawbacks associated to this strategy, the possible decrease of the biological activity of the drug when covalently attached to the MNP, and the relatively high local temperatures $\left(80-90{ }^{\circ} \mathrm{C}\right)$ that must be reached in order to effectively break the bonds, must be taken into account. The DA reaction is a thermo-reversible cycloaddition between a diene and a dienophile to form a six-membered carbocycle, using furan and maleimide moieties for instance [38]. The retro DA reaction (rDA) is a suitable release method, although a high local temperature is needed to achieve it. The possibility to use this kind of reactions to liberate molecules was first proposed by N'Guyen et al. [40].

A versatile ligand comprising different moieties was synthesized: it contained a phosphonic acid group that could be bound to the iron oxide surface of MNP; further it carried two orthogonal clickable groups, namely an alkyne moiety and a furan ring. The alkyne group was further reacted with azide end-functionalized poly(ethylene glycol) (PEG) through 1,3 dipolar cycloaddition in order to transfer the MNPs into water and confer biocompatibility. On the other hand, the furan ring was used to link a maleimide-functionalized cargo through reversible DA chemistry. In this case tetramethylrhodamine-5-C2- maleimide was conjugated as a model drug. Upon AMF exposure $(332.5 \mathrm{kHz}, 11.3$ $\mathrm{kA} / \mathrm{m}$ ), the rhodamine (Rho) was released from the MNPs through a rDA process, without any significant heating of the medium. More recently, the possibility to release DOX from MNPs based on the rDA reaction has been also reported [39]. To this end, $\mathrm{Zn}_{0.4} \mathrm{Co}_{0.6} \mathrm{Fe}_{2} \mathrm{O}_{4} @$ $\mathrm{Zn}_{0.4} \mathrm{Mn}_{0.6} \mathrm{Fe}_{2} \mathrm{O}_{4}$ core-shell MNPs were modified with a furan moiety, mixed with a maleimide-containing molecule through DA reaction, and the DOX was attached via amide linkage. It was demonstrated that under an AMF the DOX could be released, however, the experiments should be performed at $50{ }^{\circ} \mathrm{C}$ to obtain an abundant release (90\%). When incubated with HepG2 cells (human hepatocellular carcinoma), under an AMF (6200 W, $1950 \mathrm{kHz}$ ) for $15 \mathrm{~min}, 20 \%$ cell viability was observed. Nevertheless, the same MNPs without DOX also caused a significant cell death under the same AMF conditions (45\% cell viability), demonstrating that the effect predominantly came from the generated heat and not from the DOX released by rDA. 
The link of toxins instead of conventional drugs has also been reported using these thermo-sensitive covalent bonds [41]. MNPs coated with maleimide-modified silica were conjugated with ansamitocin derivatives bearing a furan group. The liberation of the ansamitocin derivative via $\mathrm{rDA}$ reaction was probed in a test tube by submitting the MNPs to an AMF, reaching a temperature of $44.1^{\circ} \mathrm{C}$ in $15 \mathrm{~min}$. After $2 \mathrm{~h}$ of applying the AMF ( $2 \mathrm{~kW}, 780 \mathrm{kHz}$ ), the supernatant was collected and used to treat Huh-7 cells (human liver carcinoma), finding that cell proliferation decreased over time when compared to non-treated groups. In vivo studies were conducted by intratumourally injecting the MNPs into tumours generated in immunodeficient mice using Huh-7 cells. Intermittent cycles of AMF were applied, so that the temperature of the tumour (measured from outside) could only rise to approximately 42 ${ }^{\circ} \mathrm{C}$. A decrease in the tumour size was observed during the first week due to increased apoptosis and reduced proliferation, although afterwards an increase in tumour volume was found. As a control, a mouse injected with the same MNPs but not subjected to the AMF was used, therefore, the solely effect of the heat generated by the AMF and the MNPs cannot be distinguished from the effect of the drug release.

Another type of thermo-responsive covalent bonds that have been used to release drugs upon AMF application are azo bonds. In a pioneering report, a PEGylated (poly(ethylene glycol)) azo-linker was used as a thermo-labile bond to functionalize MNPs, where DOX was covalently attached to the azo linker [37]. PEG molecules of different lengths were used as spacers. The MNPs were subjected to an AMF for $1 \mathrm{~h}(334.5 \mathrm{kHz}, 13.5 \mathrm{kA} / \mathrm{m})$ finding that for the shorter PEG (500 Da molecular weight) about $36 \%$ of the drug was released, while for the longer PEG (6000 Da molecular weight) only $15 \%$ was liberated. Yoo et al. went a step further by engineering MNPs that could promote thermoresistance-free apoptosis, what they coined as resistance-free apoptosis-inducing magnetic nanoparticle (RAIN) [36]. It is widely known that Heat Shock Proteins (HSPs) can shield cells from apoptosis, and therefore are involved in the acquisition of tumoural thermoresistance [42]. The RAIN consisted of an inhibitor of HSPs (geldanamycin, GM) linked via an azo linker to zinc-doped iron oxide MNPs $\left(\mathrm{Zn}_{0.4} \mathrm{Fe}_{2.6} \mathrm{O}_{4}\right)$. After $1 \mathrm{~h}$ under AMF application $(500 \mathrm{kHz}, 37.4$ $\mathrm{kA} / \mathrm{m}$ ) at $43{ }^{\circ} \mathrm{C}$, a complete release of GM was found, whereas only $7 \%$ was released when using a water bath at the same temperature. When RAIN were incubated with MDA-MB-231 breast cancer cells, $100 \%$ of death could be found due to apoptosis after 60 min upon AMF treatment, while only $25 \%$ of cells died when using MNPs not bearing GM. Interestingly, HSP90 expression levels were lower in RAINtreated cells, demonstrating that the use of a HSP inhibitor makes cells more sensitive to apoptosis. Furthermore, RAIN were intratumourally injected in mice bearing MDA-MB-231 xenograft tumours and exposed to the AMF for $30 \mathrm{~min}$. Tumour volume changes were monitored during 14 days, finding that RAIN-treated tumours were completely suppressed at day 8 .

\subsection{Caused by the matrix transformation}

\subsubsection{Polymers}

The combination of MNPs and polymers is not a recent issue. These macromolecules have been commonly used for the stabilization of MNPs in aqueous media, especially for biological applications [43]. For magnetic hyperthermia-based drug delivery, the use of thermoresponsive polymers is frequent. These polymers change their structure or miscibility when certain temperature is reached. The temperature at which a polymer undergoes a reversible change of phase is called "lower critical solution temperature" (LCST). A biocompatible polymer with a LCST slightly above the body temperature is quite interesting, as it can be exploited to load and release a drug at convenience. Different polymers with these characteristics can be found in the literature, including poly-lactic acid (PLA), poly(ethylene glycol) ethyl ether methacrylate-copoly(ethylene glycol) methyl ether methacrylate,
Pluronic $®$ F-127 (F127), poly(methylmethacrylate) (PMMA) or poly$n$-isopropylacrylamide (PNIPAM) among others [44].

As we will present along this section, MNPs have been widely used as heat source for the polymer change of phase. Different approaches have been explored for the development of a polymer-MNPs system. The MNPs can be found coated with or embedded in the thermoresponsive material (Fig. 4 ).

First, a widely utilized arrangement is represented by polymercoated MNPs. In this strategy, the MNPs are covered with a thermoresponsive and biocompatible polymer, which can simultaneously be loaded with a therapeutic molecule [45-48].

Ding and co-workers functionalized magnetic nanocubes with a thermo-sensitive sol-gel block copolymer [45]. Folic acid-decorated F127 gels were used as the coating agent. F127 is an amphiphilic block copolymer with an excellent biocompatibility. It interacts with the nanocubes and allows the loading of paclitaxel (PTX), the anticancer drug used in this work. The heating efficiency of the nanosystem was measured at different conditions of frequency and current strength, as well as at different concentrations of the sample. An increase of up to $22^{\circ} \mathrm{C}$ in $10 \mathrm{~min}$ was achieved at $2.0 \mathrm{mg} / \mathrm{mL}(200 \mathrm{kHz}, 300 \mathrm{~A})$ with an optimized drug loading - 53\% drug loading efficiency and 9.58\% drug loading content using a ratio PTX: MNPs $=2: 10(w / w)$. The optimized system was further used to monitor the release of the drug in time, with and without the application of an AMF (Fig. 5). A negligible drug release was observed when the AMF was off $\left(<10 \%\right.$ in $24 \mathrm{~h}$, at $\left.37{ }^{\circ} \mathrm{C}\right)$; conversely, a rapid release occurred when the field was turned on (40.3\% in 10 min, 93.4\% after $2 \mathrm{~h}$ of AMF application). These results also showed that the system could be precisely controlled. When a repeated AMF on-off sequence was applied, the release of the loaded drug stopped when the AMF was off, due to the return of F127 to solid phase. The cytotoxicity of the nanoconstructs - both drug-free and loaded - was evaluated in vitro in HeLa cells, with and without AMF application. Under no magnetic stimulus, both nanosystems had good cytocompatibility, with low percentages of cell death. When the AMF was applied, they showed a time-dependent death. Free nanocubes induced $70 \%$ cell death in 50 min of hyperthermia time, while cells treated with PTX-loaded nanocubes showed almost $90 \%$ reduction of their viability with the same hyperthermia time.

MNPs-embedding polymers are different from the previous examples because here the system consists of a polymeric nanoparticle in which both MNPs and the drug are inserted. Whereas in the previous approaches each nanoparticle was covered by the polymer, in here the polymer is the main entity, and it contains several MNPs as well as the drugs [49-53]. Hydrogels can be included into this category. They consist of a three-dimensional polymeric structure mainly composed by water, but insoluble due to the cross-linking [54].

\section{Combined polymers and magnetic nanoparticles}
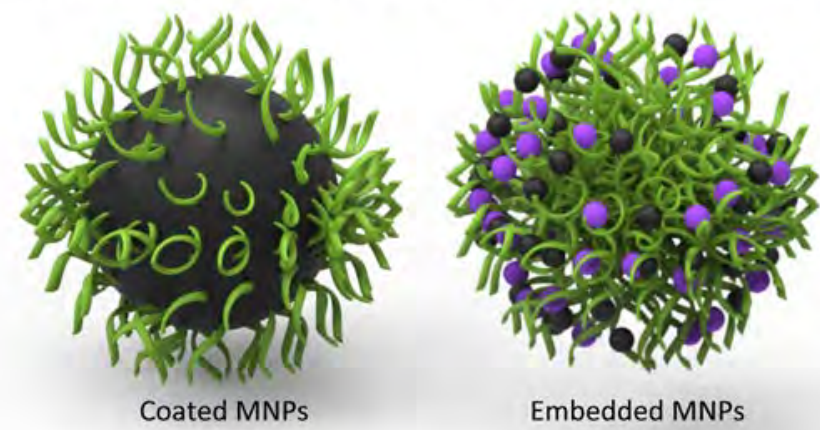

Embedded MNPs

Fig. 4. Schematic representation of the two different approaches generally used for the development or combined polymer and MNPs systems for drug release using magnetic hyperthermia. (left) Coated MNPs and (right) MNPs embedded in the thermoresponsive material. 

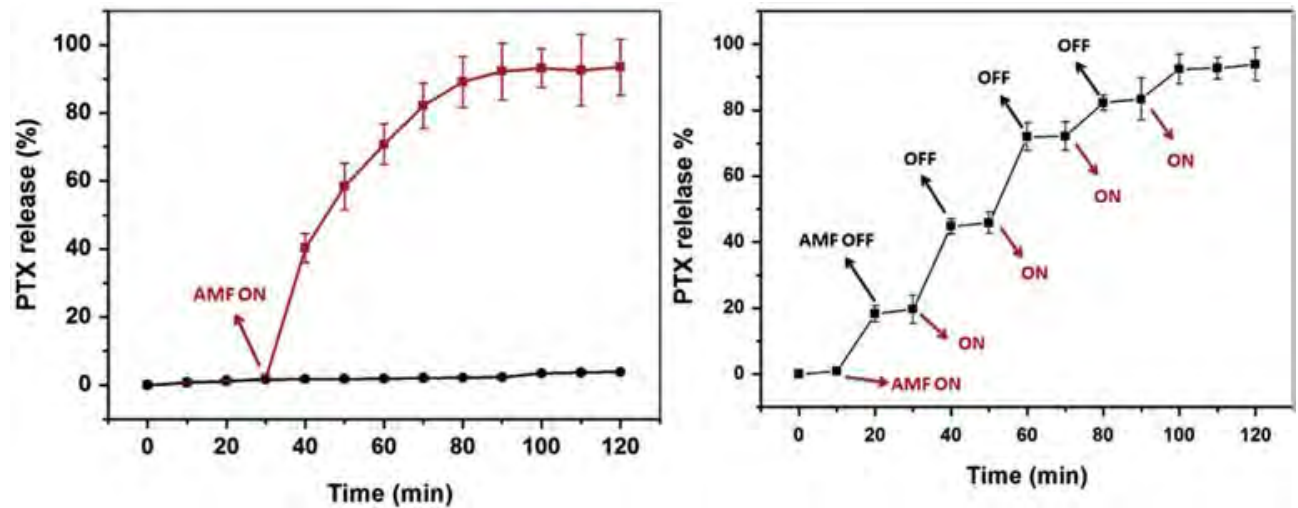

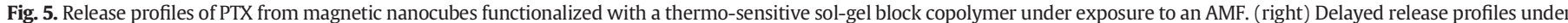

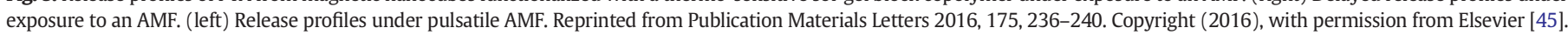

A clear example of this approach can be found in the work of Cazares-Cortes and co-workers. They developed a pH-responsive and thermo-responsive polymer-based nanogel loaded both with MNPs and an anticancer drug, DOX (Fig. 6) [50]. The gel was comprised by oligo(ethylene glycol) methyl ether methacrylate and was prepared by radical copolymerization. To obtain magnetic nanogels, $11.5 \mathrm{~nm}$ iron oxide MNPs were further encapsulated in the gel at different MNP:nanogel ratios (between 9 and $66.7 \mathrm{wt} \%$ ), showing optimal stability and homogenous distribution of the MNPs in the polymeric structure at MNP mass ratios lower than $50 \mathrm{wt} \%$ (as revealed by dynamic light scattering, DLS, and transmission electron microscopy, TEM). Afterwards, DOX was encapsulated in the magnetic nanogels. The temperature and $\mathrm{pH}$ dependence of the system was firstly studied by DLS. Changes in the hydrodynamic diameter were observed when temperature or $\mathrm{pH}$ varied, reversibly changing from a swollen to a collapsed state. Both low $\mathrm{pH}$ and high temperature induced a collapsed state. At the same temperature, nanogels had a $300 \mathrm{~nm}$ difference in hydrodynamic diameter at different $\mathrm{pH}$ values $(650 \mathrm{~nm}$ at $\mathrm{pH} 7.5$ and $350 \mathrm{~nm}$ at $\mathrm{pH}$ 5.5). Besides, $\mathrm{pH}$ influenced the collapse temperature $\left(54{ }^{\circ} \mathrm{C}\right.$ at
$\mathrm{pH} 7.5,30{ }^{\circ} \mathrm{C}$ at $\mathrm{pH}$ 5.5). The rest of the studies were performed using the $37.5 \mathrm{wt} \%$ magnetic nanogels, as this value represented the best compromise between MNP content and stability. The DOX release was measured with and without the application of an AMF ( $335 \mathrm{kHz}, 12.0 \mathrm{kA} / \mathrm{m}$, 30 min pulses) at $\mathrm{pH} 5.0$ and 7.5. After $4 \mathrm{~h}$, the release was $96 \%$ and $24 \%$ respectively without the application of the AMF. When the AMF was applied, the release was considerably accelerated, achieving $100 \%$ and $47 \%$, respectively. At $\mathrm{pH} 7.5$, the release doubled its value thanks to the AMF. The authors performed cytotoxicity studies of the nanogels using PC-3 cell lines, derived from human prostate. The cell viability was not affected by the magnetic nanogels without DOX, neither with nor without $\operatorname{AMF}(471 \mathrm{kHz}, 14.4 \mathrm{kA} / \mathrm{m}, 2 \mathrm{~h}$ ), indicating that hyperthermia itself does not produce a direct negative effect on cells. The half-maximal inhibitory concentrations $\mathrm{IC}_{50}$ were 26,19 and $8 \mu \mathrm{M}$ for the free drug, nanogel alone and nanogel + AMF (same conditions as before), respectively, proving the enhancement of anticancer effect of DOX with this system. The authors further assessed the intracellular localization of the DOX by confocal microscopy, showing that nanogels increased the amount of DOX present in the nuclei for the same drug concentration as DOX
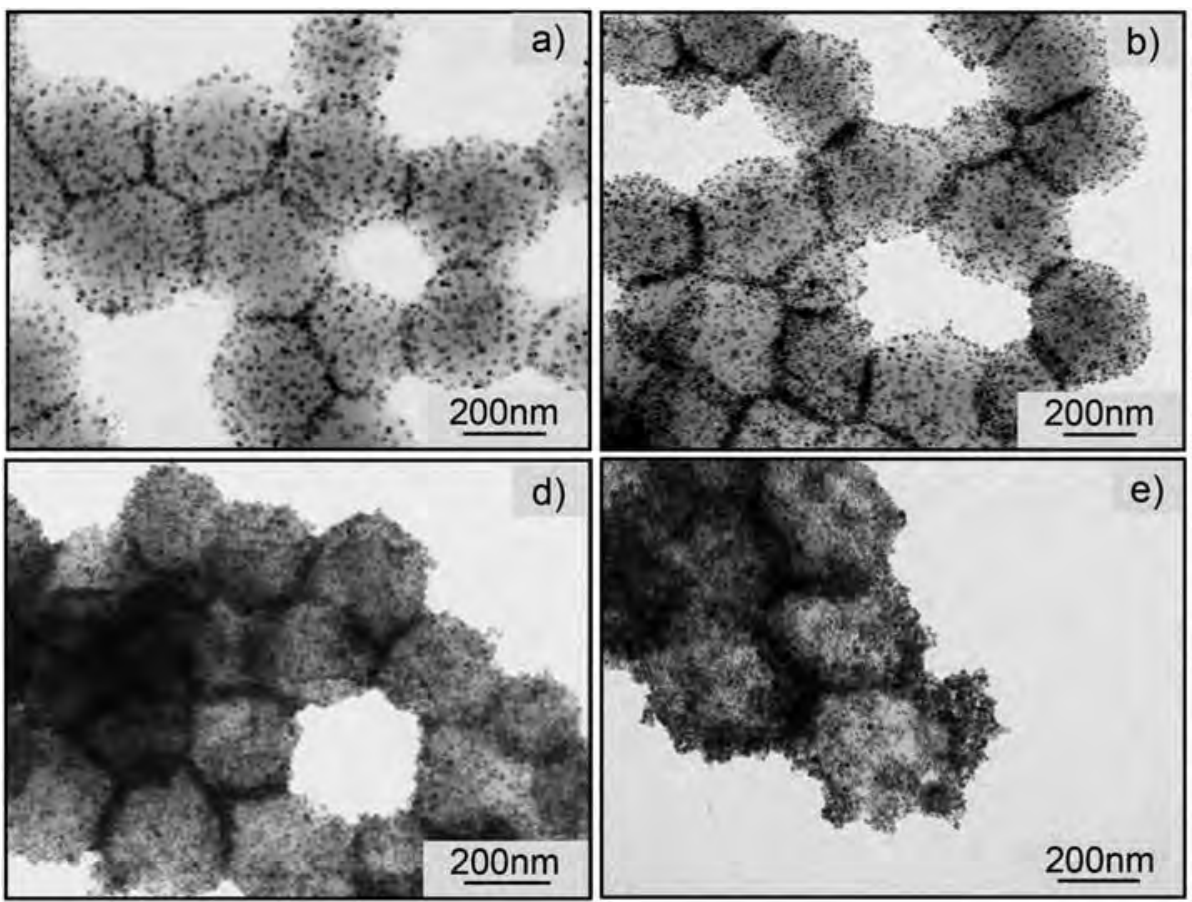

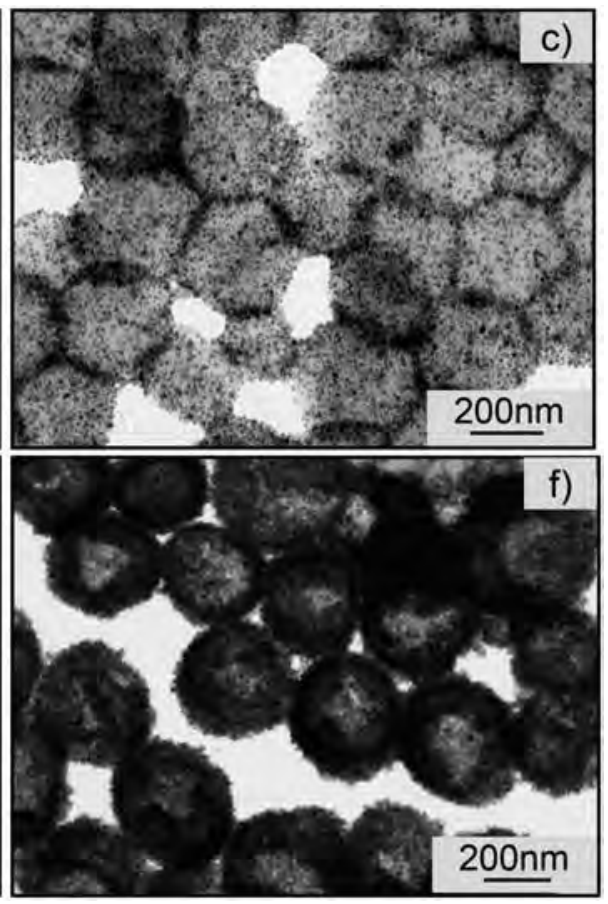

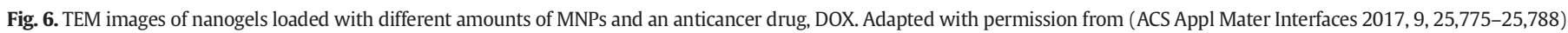
Copyright (2017) American Chemical Society [50]. 
alone: cells treated with nanogels and exposed to the AMF revealed a higher DOX fluorescence signal intensity in the nuclei than those treated without the AMF, which, in turn, showed higher DOX nuclear fluorescence than cells treated with the free drug.

As shown above, test tube and in vitro trials reflect the potential of the thermo-sensitive polymers-MNPs combination as drug delivery and cancer therapy agents. Different configurations show highly controllable systems with variable efficiencies and release kinetics (from minutes to days). This last facet can become a great advantage, as it allows the release time to be extremely tunable, using the system that best adapts to the treatment needs.

After the promising results in vitro, the therapeutic systems must be assessed in vivo before entering clinical trials. Several studies reporting in vivo experiments confirm the ability of these magnetopolymeric entities to eradicate a tumour completely (Fig. 7). In most cases, they also prevent the tumour to appear again weeks later. It is the case of magnetic nanoclusters coated with carboxylic polypyrrole loaded with DOX and further functionalized with folic acid [46]. This polymer had a LCST of around $44^{\circ} \mathrm{C}$. At that temperature, it could release $>60 \%$ of DOX in 20 min, a value that reached $90 \%$ at $46{ }^{\circ} \mathrm{C}$. The polymer coated MNPs were tested in immunodeficient female CB17 mice inoculated with human multiple myeloma cells. The animals were divided in six groups to compare the different treatment modalities: no treatment, nanoparticles without AMF, AMF (in all cases $230 \mathrm{kHz}, 8 \mathrm{kA} / \mathrm{m}$, $20 \mathrm{~min}$ ) without nanoparticles, chemotherapy alone, magnetic hyperthermia alone, and the combination. Mice treated with the whole system (loaded polymeric MNPs under AMF) showed a survival rate of $100 \% 200$ days after treatment. Meanwhile, all the other groups had a $0 \%$ survival rate after 120 days. Besides, the complete treatment succeeded in the complete removal of the tumour only 8 days after AMF exposure with no regression, while the other systems could reduce its volume, but not cure it. Of note, no toxicity of the nanoparticles was detected.

The following examples are remarkable as they prove the efficacy of nanotechnologically driven drug release with thermo-sensitive polymers against resistant tumours. Many cancer types, although they initially respond to treatment, end up by developing resistance to the drug. In such cases, elevating the dose might result in both increased toxicity and low effectiveness [55]. Magnetic nanoformulations allow the specific delivery and controlled release of the drugs. Furthermore, the heat produced by the MNPs enhances the effect of the chemotherapy, sensitizing resistant cells, as shown in many other studies [56-58].

Tumour necrosis factor (TNF)-related apoptosis-inducing ligand (TRAIL) is a cytokine that selectively induces apoptosis when it binds to DR4 and DR5 receptors, over-expressed in many cancer cells. Thus,

\section{No treatment}

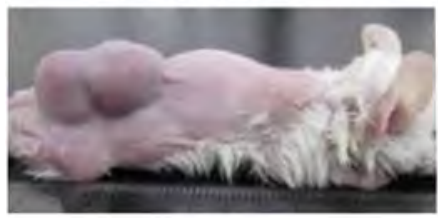

MHT

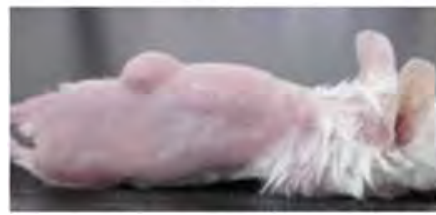

Fig. 7. Synergistic effect between chemotherapy and MHT. Photographs of non-treated mice, mice treated with chemotherapy, mice treated with MHT, and mice treated with the combination of MHT and chemotherapy 45 days after treatment. Adapted from Theranostics 2014, 4, 834-844. [46].
TRAIL or agonist antibodies (i.e. mapatumumab) were proposed to treat certain cancers [59]. However, some common malignant tumours are TRAIL-resistant, namely melanoma, glioblastoma or pancreatic cancer. In this context, Zhang and Song synthesized a negatively charged poly(organophosphazene) (PPZ) that allows the electrostatic attachment of the positively charged TRAIL. This work is as well a representative exhibition of the use of doped iron oxide nanoparticles, as they used $\mathrm{Zn}_{0.47} \mathrm{Mn}_{0.53} \mathrm{Fe}_{2} \mathrm{O}_{4}$ nanoparticles embedded in the polymer to obtain a magnetic nanohydrogel [53]. The effect of the treatment was assessed in vivo using BALB/c nude mouse bearing U-87 MG tumours (TRAIL-resistant human glioblastoma cells). The authors followed the tumour volume evolution after 1 or 2 cycles of hyperthermia, maintained around $43{ }^{\circ} \mathrm{C}$ for $1 \mathrm{~h}$ by applying an AMF of $13.3 \mathrm{kA} / \mathrm{m}$ and $366 \mathrm{kHz}$. AMF applications were carried out at day 1 and 3 post-injection. A small tumour reduction was observed in nanohydrogels alone-treated mice compared to control $\left(\mathrm{V} / \mathrm{V}_{\text {initial }}=13.6\right.$ and 15.3 respectively at day 25 post-injection), probably due to a slow but sustained TRAIL release. When a single hyperthermia cycle was applied the $\mathrm{V} / \mathrm{V}_{\text {initial }}$ ratio reduced to 6.9 , and to 1.3 with the 2 cycles treatment. The last reduction was attributed by the authors to an additional release of TRAIL from the nanosystem. Although no complete tumour regression was obtained, a clear effect of resistance reduction was achieved thanks to the combinational treatment and drug delivery system.

The last example illustrates a more complex formulation able to transport two different drugs simultaneously and release them at distinct times. It is a representative case of multidrug delivery, which can be very promising, as the combination of two different anticancer agents can avoid the development of multidrug resistance (MDR) cancers [60]. In this very complete work, Xie et al. presented a selfhealing thermo-sensitive magnetic hydrogel that allows the asynchronous release of DOX and docetaxel (DTX) [61]. The asynchronous release of both therapeutic molecules might be a potential way to overcome the MDR issue. The whole construct consist of three components: (1) DOX, (2) DTX-loaded poly(lactic-co-glycolic acid) nanoparticles (DTX/PLGA NPs) and (3) DF-PEG-DF modified iron oxide nanoparticles (PEG-MNPs, DF = 4-Formyl-benzamide). Those three elements are embedded in a chitosan/DF-PEG-DF matrix. DF groups interact with the amine of the chitosan, giving place to the cross-linking. The system is denoted as DDMH (dual-drug-loaded magnetic hydrogel). The interesting point of this approach is that, after $24 \mathrm{~h}$ of incubation at $37^{\circ} \mathrm{C}$, no DTX was released whereas $>10 \%$ of DOX did. It has to be noted that the release assays lasted up to one month (when $\sim 80 \%$ of release of both drugs is achieved). The low rate of release can be advantageous for treatments that require longer periods of drug administration. Moreover, the response to the application of an $\operatorname{AMF}(282 \mathrm{kHz}, 19.99 \mathrm{kA} / \mathrm{m})$ was also different for each drug. While DOX release was barely increased, DTX release became significantly faster using magnetic hyperthermia. This difference provides the possibility to regulate and control the co-delivery process of both drugs. In cytotoxicity assays with triple negative breast cancer cells (MDA-MB-231), the system showed a time-dependent response, and a clear synergistic effect of both drugs compared with their administration alone. After $48 \mathrm{~h}$ incubation, the viability was $19.6 \%$ if no AMF was applied, and $5.6 \%$ with the application of AMF. The system was also tested in vivo, with MDA-MB-231 tumourbearing nude mice. An intratumoural injection of saline and DDMH was performed, and after $24 \mathrm{~h}$, mice were exposed to an AMF ( $282 \mathrm{kHz}, 19.99 \mathrm{kA} / \mathrm{m}, 10 \mathrm{~min}$ ). In DDMH injected tumours, the temperature reached $48^{\circ} \mathrm{C}$, whereas in saline injected tumours only a $1.3^{\circ} \mathrm{C}$ increase was reached compared to body temperature. With a single injection, DOX hydrogel and DTX/PLGA NPs separately inhibited only partially the tumour growth compared with saline. The combined DOX and DTX/PLGA NPs hydrogel achieved much better results. However, the best outcome came from the DDMH when exposed to AMF. That was probably due to the enhanced drug delivery as well as a possible synergy of chemo- and hyperthermia therapy. Finally, toxicology of DDMH was assessed (biochemistry, hematology and histological 
analysis), and all values were within normal ranges, concluding that this nanosystem presents no obvious toxicity.

\subsubsection{Liposomes}

Since their discovery in the 1960 s by A.D. Bangham, liposomes have brought about a major revolution in the field of drug delivery. Liposomes are very simple structures based on natural or synthetic phospholipids, amphiphilic molecules that in the presence of an aqueous medium orient in a spontaneous manner to avoid the interaction of their apolar regions with water. Subsequently, a double-walled hollow sphere with an aqueous core is formed. These particular conformations exhibit unique properties that can be very useful for drug delivery applications. First of all, they present high biocompatibility due to the low reactivity of lipids. Secondly, their particular compartmentalisation allows the encapsulation of both hydrophobic and hydrophilic chemotherapeutical agents. Thirdly, liposomes can be synthetized with range of sizes between 50 and $450 \mathrm{~nm}$ for promoting the passive targeting trough enhanced permeability and retention (EPR) effect in tumours [62]. Due to all of these advantages, liposomes have been the first nanostructured device to be approved for the delivery of drugs for human use by the FDA.

In this regard, in 1988 Cuyper et al. described for the first time the encapsulation of MNPs of $14 \mathrm{~nm}$ inside of a lipid bilayer, coining the term "magnetoliposomes" [63]. Since then, the combination of MNPs with liposomes in single nanoplatforms has acquired great relevance for the development of multifunctional therapeutic devices. Due to the singular properties of MNPs, magnetoliposomes could overcome the limitations of traditional liposomes in drug delivery applications. First, in terms of selectivity, MNPs provide magnetic guidance for in vivo tumour targeting. García-Jimeno et al. demonstrated the preferential accumulation in the target tissue of magnetoliposomes intravenously administered in mice after the application of an external magnet, reducing at the same time the biodistribution in other organs related with the clearance from the blood by the immunological system as the liver or spleen [64]. Furthermore, the functionalization of liposomes with MNPs increased their colloidal stability, while also limiting the passive release of the drugs loaded $[65,66]$. Finally, the release of the drug at the target site can be triggered by the heating properties of MNPs in the presence of an electromagnetic field and therefore, improve the therapeutic efficiency [67]. Magnetoliposomes can suffer changes in their structure and permeability and release their payload in a controllable way. This change of permeability is linked to the melting temperature (Tm) of the lipid bilayer, at which there is a transition from the gel to the fluid phase of the bilayer, resulting in the subsequent diffusion of the drug. At this point it is important to remark that the composition of the lipid bilayer plays a key role and lipids with Tm above the physiological temperature $\left(37^{\circ} \mathrm{C}\right)$ are needed to avoid passive release [68]. Magnetoliposomes with higher Tm will present slower and inefficient release but this can be compensated by a good MNP loading and heating efficiency. For instance, dipalmitoyl-sn-glycerophosphocholine (DPPC, $\mathrm{Tm}$ of $41.5{ }^{\circ} \mathrm{C}$ ), is one of the most reported lipids used on thermoresponsive liposomes because it presents a melting temperature close to the clinically relevant hyperthermia values. In addition, surface grafting of magnetoliposomes with polymers, for example PEG, allows to change the gel-fluid phase transition and opens the possibility to develop new more versatile formulations [69].

Magnetoliposomes can acquire three different structures depending on the localization of the MNPs: inside the core, embedded in the bilayer or attached to the surface (Fig. 8).

The difference in the formation of each of these structures lies in the size and coating of the MNPs. In the case of the core type magnetoliposomes the limiting factors are the size of the MNPs - their diameter has to be lower than the one of the liposome core - and their coating, which must be hydrophilic, while in the case of the liposomes with the MNPs embedded in the bilayer, the MNPs have to be hydrophobic and smaller than the thickness of the lipid bilayer $(<6.5 \mathrm{~nm})$
[70]. For the surface decorated structure, hydrophilic MNPs interact mainly through electrostatic interactions and their maximum size is limited to $20 \mathrm{~nm}$ in diameter, as otherwise the liposome can be disrupted and form a bilayer around the MNP [71,72].

We should also mention that the type of structure influences the magnetoliposome functionality with respect to drug delivery. In this regard, the localization of MNPs embedded on the lipid bilayer or attached to the surface have the advantage of a direct transfer of the heat released by the MNPs to the lipid bilayer, with a more efficient controlled release. This effect was described by Amstad et al. who observed that iron oxide nanoparticles embedded in the lipid bilayer induced an increase of the permeability without disruption of the liposome after the application of a high frequency magnetic field [73]. Nevertheless, in the case of core-loaded magnetoliposomes the increase of temperature at the core has to reach the lipid bilayer and this process can be hampered by fast heat dissipation in the aqueous liposome core [74]. However, it has been demonstrated that the thermally stimulated lipid phase transition is not the only mechanism that can induce the drug release. For example, Nappini et al. suggested for the first time that nanoparticle motions in the liposome core were the main mechanism of drug release in the presence of low frequency AMF as a consequence of the alteration of the bilayer permeability. The higher release was described at a field frequency of $5.82 \mathrm{kHz}, 79.77 \mathrm{kA} / \mathrm{m}$ and at an exposure time of $50 \mathrm{~min}$, enhancing the hypothesis of the shaking effect of the nanoparticles [75]. Later on, Chen et al. described for the first time the synthesis of bilayer-decorated magnetoliposomes and their stimuli responsive release properties [76]. They observed that after radio frequency heating at $2.1 \mathrm{kA} / \mathrm{m}$ and $281 \mathrm{kHz}$, some structural changes in the magnetoliposomes appeared because of the fusion of neighbour lipid bilayers. They could demonstrate that radio frequency heating induced both permeabilization and partial disruption of the bilayer. Even more, Podaru et al. reported the release of drug from core-loaded magnetoliposomes based on short magnetic pulses $(214.8 \mathrm{~Hz}, 2393$ $\mathrm{kA} / \mathrm{m}$ ) that induced a mechanical motion of the MNPs without a significant increase in local temperature. This motion generates ultrasounds that have an effect on the stability of the bilayer and promote the release of the liposomes' cargo [77].

Finally, well-defined drug released kinetics are mandatory for a future clinical application. In this sense, the encapsulation efficiency of MNPs in the magnetoliposomes, which is dependent on their size, coating and stability, plays a key role. Shaghasemi et al. reported an exhaustive study of the parameters that can influence the heat-induced controlled release of drugs [68]. Different PEGylated magnetoliposomes of around $50 \mathrm{~nm}$ in diameter composed of four different phosphocholine lipids with different $\mathrm{Tm}$ and with iron oxide nanoparticles of $3.9 \mathrm{~nm}$ in diameter incorporated in the membrane were analysed. It was demonstrated that the concentration of MNPs in the membrane had an effect on the load release by exposure to an AMF. The higher the weight fraction of nanoparticles, the higher were the rate of release and the total amount released. However, weight fractions above $4 \%$ showed a lower stability and higher passive release (in the absence of an AMF) due to the formation of MNP aggregates in the membrane. In addition, it was demonstrated that the release kinetics was linked with the Tm of the lipid composition. In the case of the magnetoliposomes with the lower lipidic Tm only one pulse of $228 \mathrm{kHz}$ and $75.5 \mathrm{kA} / \mathrm{m}$ for $4 \mathrm{~min}$ was enough to reach the maximum release, while for the ones with higher Tm an increase in time exposure to the AMF until 8 min was necessary to observe the same release using the same AMF conditions.

Many in vitro studies have been published up-to-date and all of them follow the same formulation analysis [66,78-82]. First of all the thermal sensitivity of the magnetoliposomes is analysed. Depending of the lipid composition and the incorporation or not of polymers, the Tm can change and the stability of the formulation can be also affected. An ideal magnetoliposome should have a very low permeability at physiological temperature, while promoting drug delivery at the designed Tm. 


\section{Magnetoliposomes}
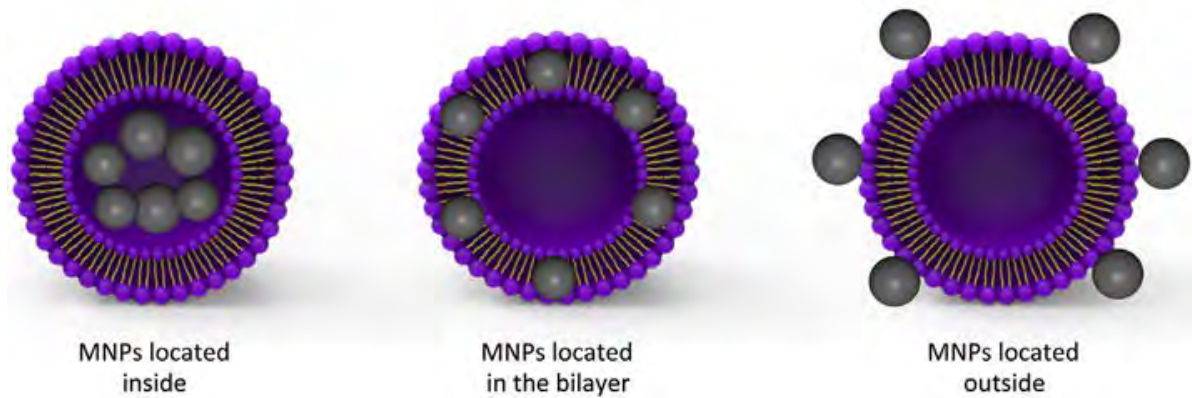

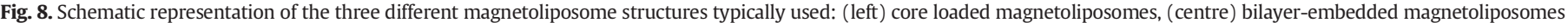
and (right) surface decorated magnetoliposomes.

Another important parameter is the drug and MNPs encapsulation efficiency to ensure that the heating produced will be enough to promote the drug release and that there is a sufficient amount of drug for a chemotherapy application. The active targeting of tumoural cells is also examined for some formulations that include targeting agents as for example folate. Finally, in vitro cytotoxicity is tested with or without AMF to check the heat-triggered chemotherapy application of the formulation.

For instance, with the objective to develop a combined thermosensitive and targeting formulation, Pradhan et al. prepared a magnetoliposome formulation with a well optimized lipidic composition of [1,2-dipalmitoyl-sn-glycero- 3-phosphocholine, cholesterol, 1, 2-distearoyl-sn-glycero-3- phosphoethanolamine- $\mathrm{N}$-[methoxy(polyethylene glycol)-2000] and 1,2-distearoyl-sn-glycero-3phosphoethanolamine- $\mathrm{N}$-[folate(polyethylene glycol)-2000] (DPPC: cholesterol:DSPE-PEG 2000 :DSPE-PEG 2000 -Folate) at 80:20:4.5:0.5 M ratio to reach a significant release of DOX at $43{ }^{\circ} \mathrm{C}$ (clinical relevant hyperthermia temperature) [78]. An encapsulation efficiency of about $85 \%$ for DOX and a temperature sensitive release of approximately $52 \%$ in $50 \%$ foetal bovine serum (FBS) at $43{ }^{\circ} \mathrm{C}$ were obtained. Worth noting, a relatively high passive release of about $17 \%$ after $1 \mathrm{~h}$ incubation in $50 \%$ FBS at $37{ }^{\circ} \mathrm{C}$ was observed. The dual active targeting induced by the folate and the MNPs in the presence of an external magnetic field was demonstrated in HeLa (human cervical carcinoma) and $\mathrm{KB}$ (human epidermoid carcinoma) cells (which overexpress folate receptors). A comparison of the system under study (MagFolDOX) with a non-targeted version without folate (MagPEGDOX), non-magnetic folate-targeted liposomes (FolDOX), non-magnetic stealth liposomes (PEGDOX) and the commercial formulation Caelyx ${ }^{\circledR}$ (DOX-loaded liposome) confirmed its higher cytotoxic effect under permanent magnetic field (Fig. 9). In addition, the combined treatment (magnetic targeting + AMF-induced hyperthermia) with MagFolDOX (30 $\mu \mathrm{M}$ DOX) improved tumour cell killing effect. KB cell viability lower than $7 \%$ after the exposure to an AMF of $12 \mathrm{kA} / \mathrm{m}$ and $290 \mathrm{kHz}$ for $1 \mathrm{~h}$ at $43.5^{\circ} \mathrm{C}$ was reached.

Despite the positive results in tumour cell death presented by Pradhan and co-workers, the high passive release observed at $37^{\circ} \mathrm{C}$ represents an important limitation for future in vivo applications. In view of this situation, two years later Kulshrestha et al., described a much simpler magnetoliposome formulation based on only two lipids: 1,2dipalmitoyl-sn-glycero-3-phosphocholine (DPPC) and 1-palmitoyl-2oleoyl-sn-glycero-3-phospho-rac-glycerol (PG) [80]. These magnetoliposomes displayed a much lower passive release at $37^{\circ} \mathrm{C}$, with a PTX release of only $1.2 \%$; at $43{ }^{\circ} \mathrm{C}$ and under AMF application $(10 \mathrm{kA} / \mathrm{m}$ and $423 \mathrm{kHz})$, the release reached $55.58 \%$. In addition, very promising results for combined hyperthermia with drug delivery were reported, with an $89 \%$ of cell death using HeLa cells.

Bilayer-embedded magnetoliposomes have also undergone in vitro studies to evaluate their potential use for in vivo experiments. Chen et al. developed a mixed formulation of magnetoliposomes containing $5 \mathrm{~nm}$ oleic acid maghemite nanoparticles but also with bare liposomes (without MNPs), both loaded with DOX $(2 \mu \mathrm{M})$ [66]. After the
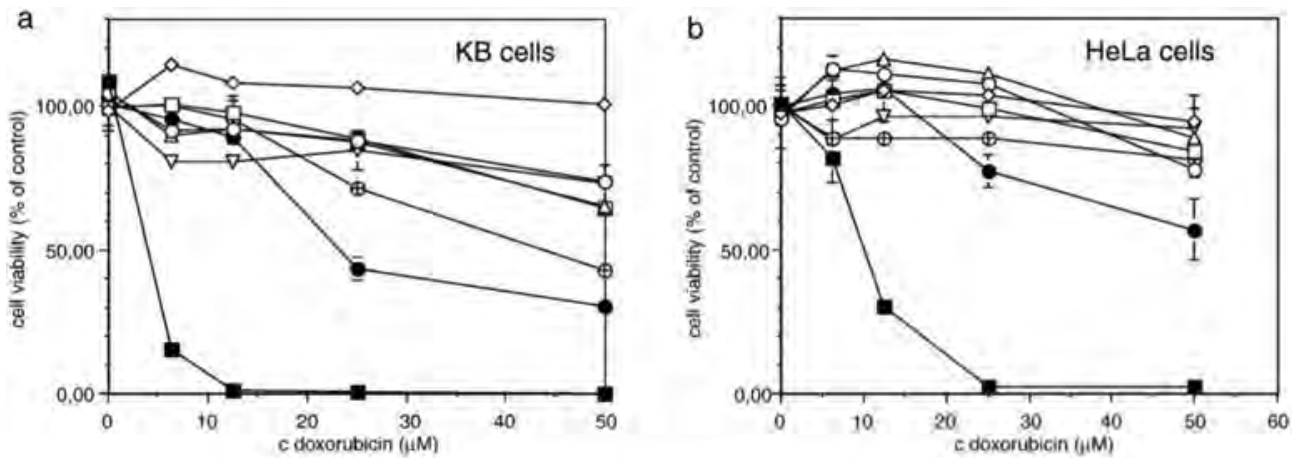

$$
\begin{aligned}
& \rightarrow-\text { free dox } \rightarrow-\text { Caelyx } \rightarrow-\text { PEGDöx } \\
& -\Delta-\text { FolDox } \rightarrow-\text { MagPEGDox }+ \text { MF } \\
& \rightarrow-\text { MagPEGDox }+ \text { MF + ImM FA } \\
& \rightarrow-\text { MagFolDox }+ \text { MF } \\
& \rightarrow-\text { MagFolDox }+ \text { MF + ImM FA }
\end{aligned}
$$

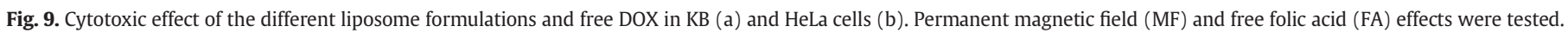
Reprinted from Publication Targeted temperature sensitive magnetic liposomes for thermo-chemotherapy. Copyright (2010), with permission from Elsevier. [78]. 
confirmation of cell uptake trough endocytosis in Huh-7 hepatocellular carcinoma cell line, cell viability studies confirmed the stability of the magnetoliposomes in terms of passive release. The DOX release was triggered with a radiofrequency electromagnetic field (field strength $7.1 \times 10^{4} \mathrm{kA} / \mathrm{ms}$ for $30 \mathrm{~min}$ ) and revealed a decrease of $40 \%$ in the cellular viability after $8 \mathrm{~h}$ and $100 \%$ after $24 \mathrm{~h}$.

Regarding the in vivo performance of these systems for cancer treatment, the first positive results based on thermo-chemotherapy were published in 2009. Yoshida et al., described for the first time the in vivo tumour regression in mouse models with human gastric MKN45 cancer cells after the intratumoural injection of docetaxel magnetoliposomes in combination with magnetic hyperthermia [83]. Tumour volume was reduced for magnetoliposomes with a DTX concentration of $56.8 \mu \mathrm{g} / \mathrm{mL}$ after the application of an AMF $(6.36 \mathrm{kA} / \mathrm{m}$ at $478 \mathrm{kHz}$ ) for $30 \mathrm{~min}$ with a power of 0.5 to $1.5 \mathrm{~kW}$ to reach a controlled tumour temperature about $42{ }^{\circ} \mathrm{C}$ for $30 \mathrm{~min}$. On the contrary, mice treated with same concentration of DTX-loaded magnetoliposomes but without AMF application, mice treated with DTX with and without AMF and mice treated with magnetoliposomes without the chemotherapeutic drug and with AMF sustained a gradual tumour growth. Furthermore, two mice (from a group of six) survived $>24$ weeks after the combined treatment of DTX-loaded magnetoliposomes and AMF but all mice died by 15 weeks after the same treatment without AMF. Since then, a few similar reports have been published, including also the combination of magnetoliposomes with photosensitizer drugs for combined MHT and photodynamic therapy [84-86]. Over the past two years the attention has been focused on the development of multifunctional magnetoliposomes with active targeting, thermo-sensitivity and imaging properties for cancer theranostics $[87,88]$. For instance, Guo et al. have recently described a multifunctional thermo-sensitive bilayer-embedded magnetoliposome that combines an active targeting (methotrexate as a surface ligand for folate receptor-overexpressed cancer cells) with DOX and a fluorescent dye (Cy5.5) for tumour imaging [88]. The intravenous injection of the system in mice bearing HeLa tumour models confirmed the tumour dual (magnetic and folate receptor) targeting efficiency over $8 \mathrm{~h}$ after the application of a magnet on the tumour region by fluorescence microscopy and MRI. Taken together with an enhanced release of DOX at the tumour site after simultaneous stimulation with an AMF (500 kHz, $20 \mathrm{kA} / \mathrm{m}, 5 \mathrm{~min}$ ) and a NIR (near infrared irradiation) laser $\left(808 \mathrm{~nm} ; 0.8 \mathrm{~W} / \mathrm{cm}^{2}\right)$ and a $100 \%$ survival rate of mice within 14 days, these results confirmed the potential of multifunctional liposomes for cancer theranostics (Fig. 10).

\subsection{Using switchable gates}

As it has been explained in the previous sections, there are many different stimuli-responsive nanomaterials that can be used to achieve a controlled drug release for improved cancer treatment purposes. All these approaches are on demand drug vehicles and can be remotely activated to deliver their cargo in the desired environment, with spatiotemporal control, only when the external stimulus is on. However, it is desirable to achieve switchable systems that could be activated when the stimulus source is on and come back to their original state when the stimulus is removed. This would facilitate a repetitive activation of the drug release once the nanomaterial has reached the tumour site. This switchability is a very promising feature for a drug delivery system as it makes possible to better control the amount of drug that is released in the tumour; furthermore, repetitive activations give the possibility to perform several cycles of drug release and tumour treatment.

In addition to some approaches based on polymeric nanostructures described in previous sections, an alternative design for switchable systems is based on a platform composed of two different materials: a core composed of a porous material with large mesoporous volume, where the therapeutic molecule is retained, and a coating that blocks the pores, acts as gatekeeper preventing the drug release in physiological conditions and is able to change its conformation in the presence of the stimulus, allowing the tailored drug release (Fig. 11). The conformation alteration should be reversible, changing from the capping state to the uncapping state when the stimulus is OFF and ON, respectively.

Among the matrix materials used for these delivery systems there is one type that is by far the most used one, namely mesoporous silica (Fig. 12) [15,16,89-95]. This material has unique characteristics that make it a good candidate for drug delivery applications, such as its high surface area, good biocompatibility, large pore volume and large a)

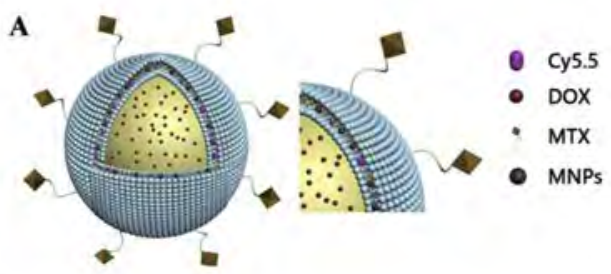

c)

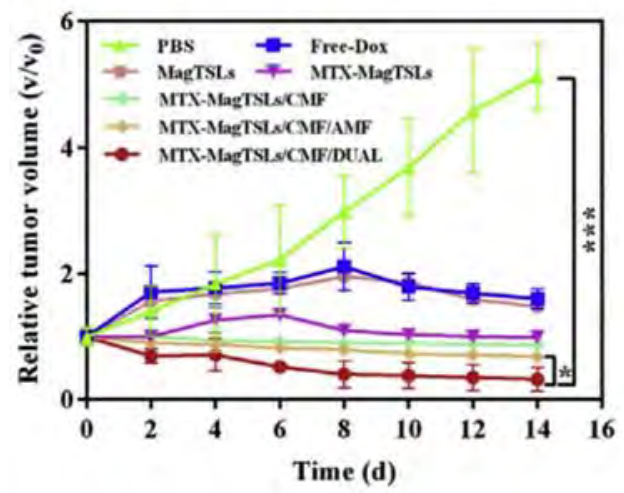

b)

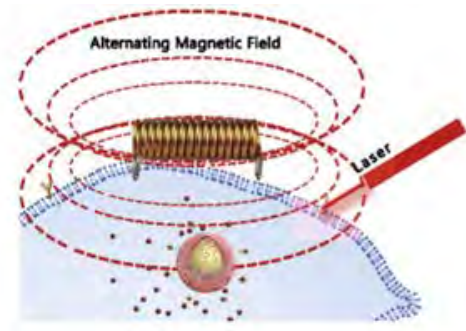

d)

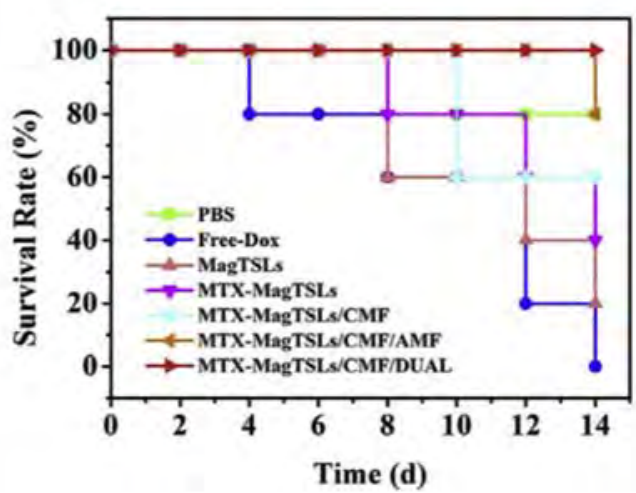

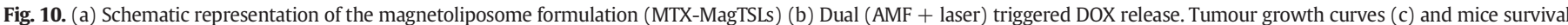

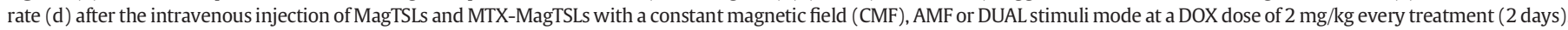

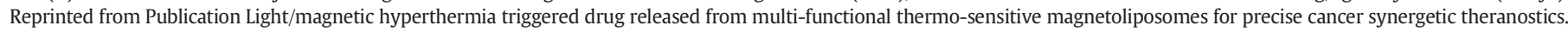
Copyright (2017), with permission from Elsevier. [88]. 
pore sizes, together with easy surface modification [96]. In general terms, the nanocarriers based on mesoporous silica nanoparticles (MSN) can be of different shapes, with the drug molecules placed within the pores. The pores are externally blocked by some cap ligands that work as gatekeepers of the drug. There are several examples of different thermo-responsive cap ligands, the most commonly used ones being phase change materials as thermo-sensitive polymers [15,90,93,95], although there are also few approaches that use small molecular nanovalves for blocking individual pores [16,91,92].

In the first part of this section, we will review the use of thermosensitive polymers as gatekeepers, a widely used strategy for drug delivery nanocarriers. Baeza et al. used the copolymer poly(ethylenimine)-bpoly(N-isopropylacrylamide (PEI/NIPAM) with a double purpose [93]. On the one hand, the polymer is responsible of blocking the pores of the mesoporous matrix avoiding/allowing the release of the model cargo molecule (fluorescein) depending on the temperature. On the other hand, the polymer is able to electrostatically retain a second model molecule, in this case a protein (Soybean Trypsin inhibitor typeII-S). Results showed that the nanocarrier was able to release the two different molecules under the application of an AMF $(24 \mathrm{kA} / \mathrm{m}$, $100 \mathrm{kHz}$ ) for $6 \mathrm{~h}$. The release of the surface-retained protein was similar to the one observed when externally heating the nanocarrier at $45^{\circ} \mathrm{C}$, but the release of the fluorescein, located inside the nanocarriers, was much faster than the one observed under external heating. Other approaches are based on the use of polymers that respond to more than one stimulus; typically one internal and one external stimulus are combined in order to ensure a better drug release. The combination of heat and $\mathrm{pH}$ stimuli is very appropriate and is being investigated for cancer treatments. Guo et al. designed a nanocarrier based on a $\mathrm{Fe}_{3} \mathrm{O}_{4}$ core and a mesoporous silica shell functionalized with the thermo-sensitive polymer poly[(ethylene glycol)-co-(L-lactide)] (P(EO-co-LLA) that has a sol-gel transition at $45^{\circ} \mathrm{C}$. The drug molecule, DOX, was loaded within the silica matrix and release experiments concluded that the best drug release profiles were achieved when submitting the nanocarrier to an AMF $(250 \mathrm{kHz})$ at a $\mathrm{pH}$ of 5.8 . In vitro experiments showed that the drug loaded nanocarriers exhibited a quite important concentrationdependent cytotoxicity effect (from $75 \%$ to about $30 \%$ of cell viability

\section{Release of therapeutic molecules using switchable gates}
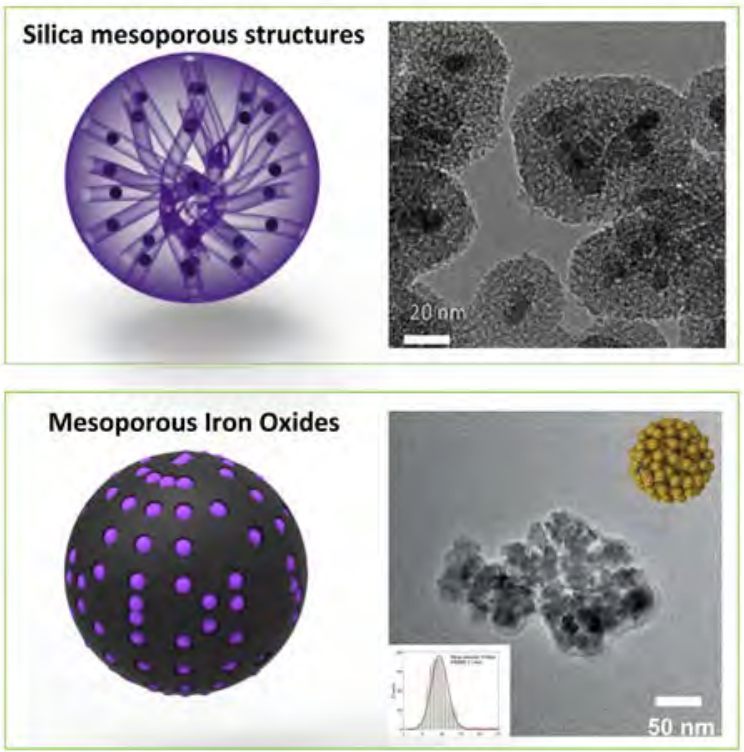

Fig. 12. Schematic representation and selected transmission electron images from Mesoporous structures used to carry therapeutic molecules in the frame of switchable systems with two different compositions: Silica and Iron Oxides. Adapted with permission from [97] Copyright (2012) American Chemical Society. Adapted from [98] Licensed Content Publisher John Wiley and Sons.

with 3.125-50 $\mu \mathrm{g} / \mathrm{mL}$ DOX, respectively) after $6 \mathrm{~h}$ of incubation. However, after the exposure to the AMF a reduction in the cell viability was observed, proving the efficacy of the nanocarrier [15]. Other type of thermo-sensitive polymers can be the temperature-degradable ones (Fig. 11). The main inconvenience of this type of polymers is that they do not fulfil the switchability requirement and although different stimuli conditions could trigger a different response in terms of amount of released drug, the polymer would never recover its physiological state

\section{Release of therapeutic molecules using switchable gates}

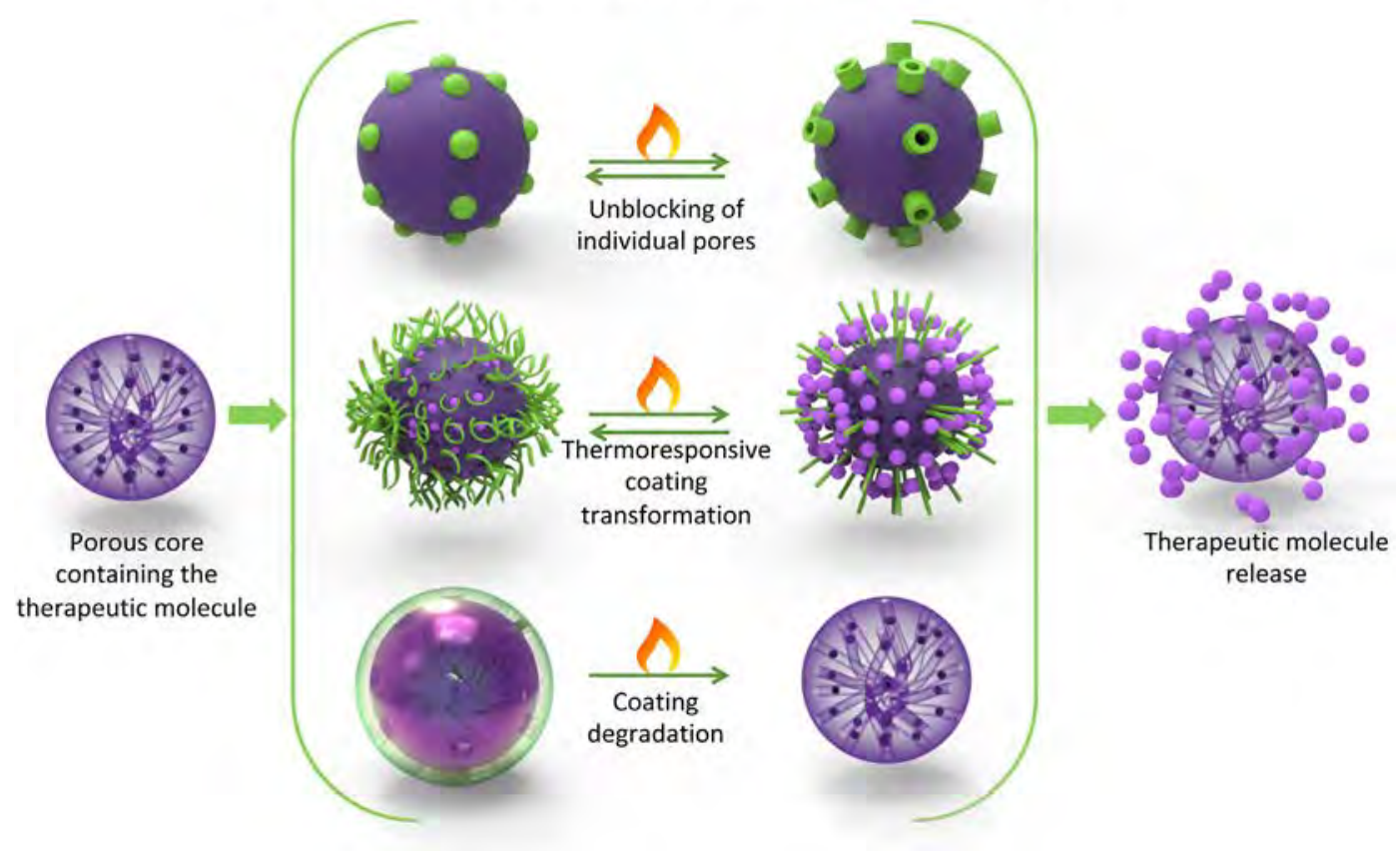

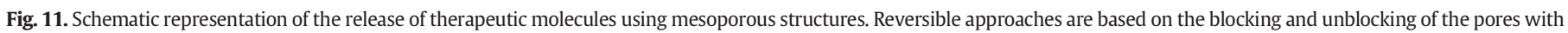

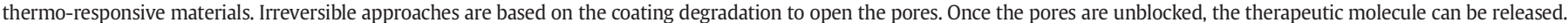


after degradation. Sain-Cricq et al. synthesized a nanocarrier consisting of magnetic core-shell $\left(\mathrm{Fe}_{3} \mathrm{O}_{4}-\mathrm{SiO}_{2}\right)$ particles functionalized with PEG through a thermo-labile azo-bond (see section 2.1. about release caused by bond break, Fig. 3) [90]. The molecule used as model was Rho 6G. The authors performed test tube release and in vitro cytotoxicity experiments, the results indicated that the nanosystem was able to release the content when exposed to an AMF even though no macroscopic temperature increase was observed and that no cytotoxicity effect was detected.

As previously mentioned, there are also a few examples in the literature that use other types of gatekeepers different from polymers. Some small molecules can be used to block individual pores. So is the case of the work of Rühle et al., who designed a nanocarrier made from MSNs functionalized with maleimide. An adamantane group was attached to the maleimide though a thermally reversible $[2+4]$ DA cycloaddition with a furan-modified linker (for more details on the DA cycloaddition, see the section about release caused by bond break). This system can undergo a cycloreversion when exposed to an external AMF $(370 \mathrm{kHz}$, $5 \mathrm{kw}, 30 \mathrm{~min}$ ), resulting in the release of its content [92]. Another approach in which nanovalves were used is the one reported by Thomas et al., who synthesized a nanocarrier consisting of MSNs whose surface was modified with pseudo-rotaxane as thermo-responsive gatekeeper [91]. This nanovalve remained closed at physiological temperature and opened when heated; release test tubes experiments demonstrated that the nanovalve was opened when the nanocarrier was submitted to 1 min of AMF even when no macroscopic temperature increase was observed. In vitro experiments performed with MDA-MB-231 cells showed that although the nanocarrier without the drug produced about $15 \%$ of cell after the exposition to the AMF $(37.4 \mathrm{kA} / \mathrm{m}, 500 \mathrm{kHz})$, the cells incubated with drug-loaded nanocarrier and submitted to the AMF increased their death rate (about 35\% cell death). More recently, Shanta Moorthy et al. have used crow ether triad (CET) molecules as gatekeepers units for a core-shell magnetic mesoporous silica drug delivery system [16]. These cap molecules were not only thermo- but also $\mathrm{pH}-$ sensitive, and release experiments showed that even though AMF $(14.3 \mathrm{kA} / \mathrm{m}, 409 \mathrm{kHz})$ exposure per se did not induce drug release, a synergistic effect between both stimuli led to good release profiles. In vitro experiments showed that the nanocarrier itself had a concentrationdependent cytotoxic effect, but when cells were exposed to AMF the cell death rates were higher for each of the nanocarrier concentrations tested (from 20 to $100 \mu \mathrm{g} / \mathrm{mL}$ ). For instance, at 60 and $80 \mu \mathrm{g} / \mathrm{mL}$ the cell death observed after the exposition to the AMF increased $20 \%$ when compared with the cells without AMF exposition.

DNA molecules have also been used as cap ligands in MSN-based drug delivery systems, taking advantage of the inner natural ability of DNA strands to reversibly hybridize with temperature (see section about release caused by bond break). Besides, it is also known that the melting temperature can be tuned by changing the ratio of CytosineGuanine ( $C-G$ ) bonds. Some representative examples of this strategy are the ones described by Ruiz Hernádez et al. and Zhu et al. In the first one, the surface of the silica particles was functionalized with a single-stranded DNA, while the complementary strand was linked to the MNPs, so once the particles were loaded with the drug molecules, hybridization of both DNA strands led to the blocking of the pores and the external exposition of the MNPs [95]. The second approach was very similar to the first one but in this case the MNPs (lacking DNA functionalization) were located inside the silica particles, together with DOX [94]. The mesoporous particles were surface-modified with a single-stranded DNA and capped with the complementary DNA strand. In both systems the drug release was tested by externally heating the nanocarriers, but not under AMF exposure. However, these results confirmed that the release of the cargo was due to a temperature increase.

The last example concerning MSN carriers is the one published by Chen et al., in which the authors designed a drug delivery system based on MSN carriers loaded with a chemotherapeutic drug, $(S)-(+)-$ camptothecin (CPT) and externally functionalized trough chemical bonds with monodisperse $\mathrm{Fe}_{3} \mathrm{O}_{4}$ particles as capping agents [89]. Release experiments results demonstrated that a repeated exposure of the nanocarriers to AMF of different durations (1, 3 and $5 \mathrm{~min}$ ) led to different drug release profiles. Furthermore, the authors estimated the number of MNPs that have been removed from the surface of the system by measuring the loss of weight after different AMF times ( 1 to $5 \mathrm{~min}$ ). The results indicated that the number of MNPs that were removed from the surface went from 2861 particles in the case of one minute exposure to 14,943 particles in the case of five minutes. In vitro experiments showed that the nanocarrier was uptaken by A549 cells and the cell viability decreased significantly after 3 min of AMF exposure only in the case of cells treated with the drug-loaded nanocarrier.

Apart from the silica mesoporous materials, there are other materials such as mesoporous iron oxide particles that possess at the same time a nanocrystalline morphology with mesoporous characteristics and good magnetic properties required for generating heat when exposed to an AMF (Fig. 12). This type of particles were used by Benyettou et al. for developing a nanocarrier system consisting of mesoporous $\gamma$ $\mathrm{Fe}_{2} \mathrm{O}_{3}$ particles with a pore size of about $8 \mathrm{~nm}$ and loaded with DOX molecules [98]. The surface of the particles was covered with a thermosensitive block copolymer based on ethylene oxide and propylene oxide, known as Pluronic ${ }^{\circledR}$ F-108, that has large changes in water content, rigidity and hydrophobicity at $41{ }^{\circ} \mathrm{C}$ with respect to its state at physiological temperature $\left(37^{\circ} \mathrm{C}\right)$. Release test tube results showed that this delivery system was also sensitive to changes in $\mathrm{pH}$, resulting in a very slow release of the DOX content under acidic conditions. On the contrary, a sudden cargo release was achieved when the nanocarrier was submitted to an AMF (26.8 kA/m, $464 \mathrm{kHz}$ ) for 10 min under the same acidic conditions, thus achieving the synergistic effect of both stimuli. Furthermore, when analysing the efficacy of the nanocarrier in vitro under exposure to the AMF it was observed that, although some unspecific DOX release promoted cell death in the absence of the AMF stimulus, the cell viability decreased considerably when cells were exposed to the AMF for one hour.

Instead of coating the iron oxide surface with the thermo-responsive polymer, Zhang and co-workers used a mesoporous iron oxide nanoparticles whose pores were filled with a mixture of a thermo-sensitive polymer (1-tetradecanol, PCM), PEG (2000) and the chemotherapeutic agent (DOX) [99]. The role of PEG in the mixture is to decrease the interfacial tension between the PCM and water, facilitating the release of DOX. In fact, the release efficiency was $>70 \%$ higher in the presence of PEG. Different PCM:PEG:DOX ratios were tested, and the chosen sample (PCM:PEG:DOX weight ratio of 160:40:1) was stable and presented a solid state in water at $37^{\circ} \mathrm{C}$, becoming soluble at $42^{\circ} \mathrm{C}$. This temperature range is optimal, as it is equivalent to body temperature. Pores of a diameter of around $2.1 \mathrm{~nm}$ could be observed in the iron oxide MNPs by TEM; the disappearance of the pores in the images of MNPs loaded with the PCM/PEG/DOX mixture, together with Fourier transform infrared spectroscopy (FT-IR) confirmed the loading of the nanosystem. Regarding the release, in the absence of an AMF, no DOX leakage was observed for 8 days at room temperature. This reflects that the drug is safely contained in the nanostructure when no stimulus is applied. After those 8 days, the system rapidly achieved $80 \%$ of DOX release in $<12 \mathrm{~h}$ when exposed to an AMF $(200 \mathrm{kHz}, 47.8 \mathrm{kA} / \mathrm{m})$.

Another recent approach where no "classical" mesoporous materials-based nanocarrier was used is the one proposed by Wang et al. The system developed consisted of a core-shell-shell structure with a cavity centre of $80 \mathrm{~nm}$ where the drug (DOX) is located, surrounded by a layer of magnetite and an outer layer of mesoporous carbon [100]. The surface was functionalized with chitosan molecules that interact both covalently and electrostatically with the mesoporous carbon layer. Release test tube experiments revealed that this coating prevents the release of the drug molecules at physiological conditions after $78 \mathrm{~h}$. Besides, the accumulation of the drug released was recorded 
for $80 \mathrm{~h}$ (serial $10 \mathrm{~h}$ treatment cycles were performed), confirming drug release during the first 30 min of AMF and only minimal drug release during the next $9.5 \mathrm{~h}$. Furthermore, in vitro experiments corroborate that the drug was released from the nanocarrier only in the presence of an AMF and a synergistic effect between drug release and the heat produced by the nanocarrier was achieved.

To date, most of the works published in this particular field were strongly focused on the design and characterization of new drug delivery systems. Almost in all of them test tube release experiments were performed for studying the behaviour of the nanocarrier under different conditions. Many of them, but not all, carry out preliminary in vitro experiments for assessing the potential cytotoxicity of the materials. This aspect is of great importance, as undesired and non-specific drug release could occur if the gatekeepers do not block completely the pores of the material, resulting in a cytotoxic effect in physiological conditions, as indeed can be seen in some works [16,98]. Furthermore, some works also tested the efficacy of the nanocarriers in vitro in different cell lines under AMF stimulus. Here it is worth mentioning that the conditions of the AMF reported in different works are very different. For instance, the exposure time can vary from $10 \mathrm{~s}$ [16] to one hour [98]. In almost all cases a decrease in cell viability after the AMF treatment is observed, indicating that different drug delivery systems can be remotely activated by an AMF.

However, there are also few works in which the in vivo effectiveness of the drug delivery systems is tested. For instance, Guisasola et al. and Wang et al. used different animal models (an allograft or a xenograft tumour model, respectively) to investigate the effectiveness of their drug delivery systems $[100,101]$. The model used by Guisasola et al. consisted of a heterotopic allograft tumour model where EL4 cells were subcutaneously injected in the flank of the animal. The model used in the work of Wang et al. was a heterotopic xenograft tumour model where C6 cells were subcutaneously injected in the flank of inmunodeficient mice. In both works the drug delivery systems were injected intratumourally and the animals were subjected to several AMF cycles (three cycles of $30 \mathrm{~min}, 105 \mathrm{kHz}$, and $18 \mathrm{kA} / \mathrm{m}$, and four cycles of 30 min of AMF using a magnetic generator $110 \mathrm{~V} 400 \mathrm{~A}$, respectively, although the AMF parameters between both works cannot be directly compared because of the different ways of providing the data). Results showed an inhibition in the tumour growth in the groups of animals that received the drug-loaded nanocarriers and were submitted to the AMF. In the case of Guisasola et al. it was observed that $48 \mathrm{~h}$ after the last AMF treatment the volume of the tumour with drug-loaded particles and AMF exposure was the half than of all the controls groups that were tested (DOX alone, AMF alone, empty nanocarriers with AMF, drug loaded nanocarriers without AMF) (Fig. 13). In the experiments carried out by Wang et al. an almost complete regression of the tumour in the group of mice that receive the complete treatment was observed in comparison with all the controls groups (AMF alone, drug loaded nanocarriers without AMF and empty nanocarriers with AMF). However, a direct comparison of the results obtained in the two studies is not straightforward, as there were substantial differences in the experimental procedures.

\subsection{Caused by matrix degradation}

As a proof of concept, it has been recently reported the possibility to design thermo-responsive nanostructures that are able to disintegrate and release molecules upon thermal triggering. Self-immolative polymers (SIPs) consisting in poly(ethyl glyoxylate) (PEtG) can be triggered to degrade upon the cleavage of a temperature-responsive end-cap based on a rDA reaction (PEtG-DA) and subsequent furan elimination [102]. To test the thermo-responsiveness, the polymer was heated at different temperatures for $24 \mathrm{~h}$, finding that $85 \%$ depolymerization occurred at $75^{\circ} \mathrm{C}$, whereas a non-responsive PEtG end-capped underwent $<10 \%$ degradation. Thermo-responsive micelles were formed using DEtG-DA and PEG, and then MNPs were incorporated and the whole system was subjected to an AMF for $3 \mathrm{~h}(10.2 \mathrm{kA} / \mathrm{m}, 755 \mathrm{kHz})$. Thermo-induced disassembly of the micelles was demonstrated but only when AMF was performed at a global temperature of $72{ }^{\circ} \mathrm{C}$; thus the translation of such nanostructures into the clinical setting is still very challenging.

\section{In situ expression of therapeutic genes}

The main objective of gene therapy is the delivery of foreign genetic material (gene, gene segments or oligonucleotides) into specific cells, with the main aim to treat or alleviate a disease [103]. In the case of tumours, this approach can be used to inactivate oncogenes, replace defective tumour suppressor genes, or more recently to transfer transgenes into cancer cells in order to restore normal function or assist in their death [104].

The possibility of controlling target gene expression in tumours in vivo in a spatiotemporal fashion is a promising strategy for gene therapy. An emerging way to remotely control gene therapy is based on the use of magnetic fields, as they can deeply penetrate into tissues [105]. Further, inducible promoters allow the expression of therapeutic genes in a controllable way. Triggering activation of genes by magnetic hyperthermia can be approached by using expression vectors containing heat-responsive promoters [106,107]. The gene products can directly kill the cells or act in a synergic way to increase the antitumoural effect of the hyperthermia. One of the first examples of gene therapy mediated by magnetic hyperthermia was described by Ito et al. in 2001 [108]. They reported the use of a combined TNF- $\alpha$ gene therapy driven by the heat-inducible promoter GADD 153 (Growth Arrest and DNA Damage) using magnetite cationic liposomes (MCLs). After injecting the MCLs with the plasmid containing the
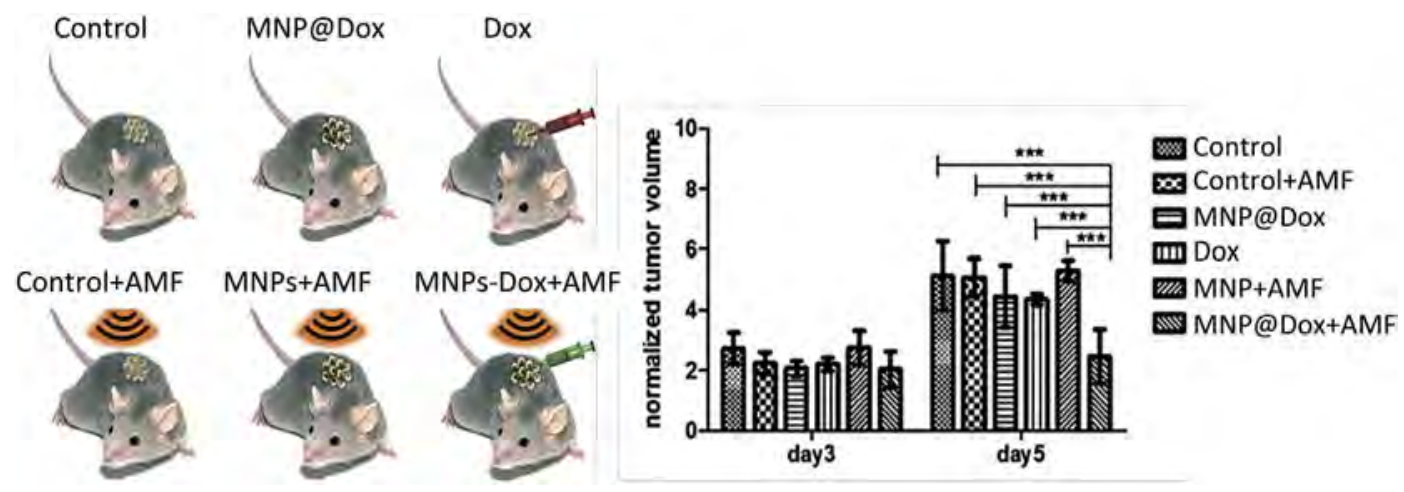

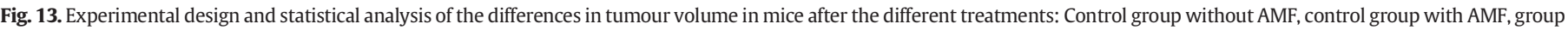

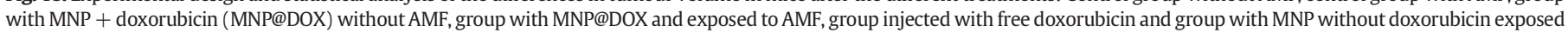

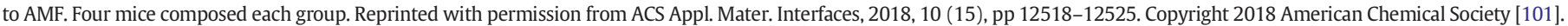


human TNF- $\alpha$ gene into subcutaneous tumours in nude mice, AMF was applied $(30.6 \mathrm{kA} / \mathrm{m}, 118 \mathrm{kHz})$ for $30 \mathrm{~min}$ and the temperature maintained at $46{ }^{\circ} \mathrm{C}$. A 3 -fold increase in TNF- $\alpha$ expression was observed in heated tumours compared to control ones, resulting in a significantly reduction in the tumour volume.

It is widely known that the major group of proteins expressed upon heat stress is represented by the HSPs, and that even mild magnetic hyperthermia is capable of giving rise to the expression of HSPs in vitro and in vivo [109]. Therefore, many inducible vectors for gene therapy activation using magnetic hyperthermia have been constructed using expression systems under the control of a HSP promoter [110]. For instance, Tang et al. reported that when injecting $\mathrm{Mn}-\mathrm{Zn}$ ferrite nanoparticles and a vector encoding $\beta$-galactosidase ( $\beta$-gal) driven by a HSP70 promoter, $\beta$-gal expression was highly increased after AMF treatment [111]. Although the vector did not encode for an anticancer molecule, the feasibility of using these inducible promoters together with magnetic hyperthermia was demonstrated. Similarly, Yamaguchi et al. explored the remote activation of the therapeutic gene TNF- $\alpha$ with MCLs and AMF using a gene expression system under the control of a HSP70B' promoter [112]. Once activated by heat, the HSPs could bind the promoter, acting as a switch to induce the expression of TNF- $\alpha$. Additionally, a tetracycline (Tet-) transactivator system was included in the plasmid, as a positive feedback loop to enhance the activity of the HSP70B' promoter. In vitro, human lung adenocarcinoma A549 cells were transfected with the construct, incubated with MCLs and subjected to AMF exposure ( $30.6 \mathrm{kA} / \mathrm{m}, 118 \mathrm{kHz}$ ) for $30 \mathrm{~min}$. The temperature was controlled at 43 or $45^{\circ} \mathrm{C}$ by adjusting the electrical power, and the TNF- $\alpha$ production and cell viability were measured. TNF- $\alpha$ production in cells transfected with the plasmid was only observed after AMF exposure, indicating that the HSP70B' promoter was activated by the temperature increase triggered by the MCLs under the AMF. Cell viability also decreased drastically in cells transfected with the therapeutic system and exposed to the AMF, when compared with cells transfected with a mock plasmid or not exposed to the AMF. In vivo, heat-induced TNF- $\alpha$ gene therapy was evaluated in mice bearing tumour xenografts. Only when the therapeutic gene was present and the animals were subjected to the AMF for $30 \mathrm{~min}$, the tumour volume diminished and a high concentration of TNF- $\alpha$ was found in them. In order to avoid the intratumoural injection and/or to overcome the limited tumour targeting ability of conventional carriers, Yin et al. proposed the use of mesenchymal stem cells (MSCs) to deliver the vector [113], as MSCs possess the intrinsic ability to migrate to tumours. The system was composed of magnetic zinc-doped iron oxide NPs coated with a silica shell (MCNP), complexed with a heat- inducible gene vector (HSP70B' promoter) encoding a secretable form of TNF-related apoptosis-inducing ligand (Fig. 14 A-D). TRAIL is a member of the TNF superfamily, which can selectively induce apoptosis through an extrinsic pathway in a broad range of human cancer cells, while sparing normal cells. Targeting the extrinsic pathway is considered a promising strategy to regulate apoptotic cell death when compared to the intrinsic one, as the later frequently gives rise to drug resistance [114]. Despite the encouraging preclinical results, its clinical translation has been limited due to its short biological half-life in vivo [115]. To overcome this limitation, the proposed MNPs had the dual purpose of enhancing the delivery of the heat-inducible plasmid to the MSCs by magnetic uptake, and of activating the production of TRAIL via mild magnetic hyperthermia. Hence, once the MNPs bearing the vector were loaded into the MSCs, these MSCs carrying the MNPs along with the vector were exposed to an AMF for $1 \mathrm{~h}(5 \mathrm{kA} / \mathrm{m}, 225 \mathrm{kHz})$, achieving a temperature of $41^{\circ} \mathrm{C}$. To corroborate that TRAIL was being secreted, this conditioned media from MSCs exposed to the hyperthermia was collected and added to ovarian cancer cells A2780 in vitro (Fig. $14 \mathrm{E}$ ). An important decrease in cell viability was found when compared to cells treated with conditioned media of MSCs not exposed to the AMF (Fig. 14 F). The activation of caspases, which are implied in apoptosis cell death, was demonstrated by qPCR (quantitative Polymerase Chain Reaction) (Fig. 14 G).
Among cancer gene therapy strategies, enzyme-prodrug therapy utilizes exogenous enzymes for the controlled conversion of prodrugs to drugs only in target cells. To this end, Nemani et al. proposed the use of alginate microcapsules containing MNPs and an engineered E. coli strain expressing cytosine deaminase upon thermal induction [116]. In this sense, E. coli was designed to overexpress the enzyme at elevated temperatures $\left(42{ }^{\circ} \mathrm{C}\right)$ when compared to $30^{\circ} \mathrm{C}$, by ligating the cytosine deaminase gene under the transcriptional control of a thermo-responsive promoter cassette. Once expressed, this enzyme is able to convert the non-toxic prodrug 5-fluorocytosine (5-FC) to the toxic metabolite 5-fluorouracil (5-FU), which inhibits cell proliferation leading to cell death [117]. After encapsulating these modified E. coli bacteria with iron oxide nanoparticles in alginate capsules, the application of an AMF (35.9 kA/m, 30 min) led to remote triggering of cytosine deaminase expression. As a proof of concept, three tumoural cell lines were incubated with the microcapsules already pre-heated at 42-43 ${ }^{\circ} \mathrm{C}$ using an external water bath or an AMF, together with the prodrug for $72 \mathrm{~h}$, and the cell viability evaluated. The cytotoxicity of the microcapsules activated by AMF was comparable to that of the microcapsules pre-heated in a bath, and to the one produced by 5 -FU by its own. On the other hand, the microcapsules without the prodrug also showed toxicity, which could be related to the presence of bacterial toxins. Although the magnetic triggering of cytosine deaminase expression was demonstrated, further studies should be performed to demonstrate the feasibility of the system once incorporated into cells.

\section{Conclusions and future remarks}

Thermo-responsive nanocarriers based on magnetic nanoparticles have remarkable potential for applications in cancer treatment. Recent developments in the synthesis and functionalization of nanoparticles led to the creation of a large toolbox for engineering the physicochemical features of the nanocarrier that enable the on-demand release of therapeutic drugs under the magnetic hyperthermia stimulus, with excellent spatiotemporal control. As highlighted in the first section of this review, drug release from thermo-responsive matrices can be achieved by a multitude of strategies, including bond breaking, matrix transformation or degradation, and the use of switchable gates. The use of MNPs also capitalizes on the synergistic effect of the combined magnetic hyperthermia - chemotherapy approach to enhance the treatment efficiency. Moreover, multifunctional nanocarriers can be designed, by introducing tumour-targeting ligands to improve their targeting capability, or specific fluorescent dyes to enable molecular imaging. By engineering the formulation of the nanocarrier, it is also possible to obtain platforms for asynchronous delivery of two different chemotherapeutics (which opens up new avenues for addressing multidrug resistance in cancer treatment), or to create dual stimuliresponsive nanocarriers. Magnetic hyperthermia has also demonstrated its potential as a promising strategy for controlling in situ expression of therapeutic genes or enzyme-mediated conversion of anticancer prodrugs into drugs.

Despite tremendous advances regarding the design and characterization of the nanocarriers, the numerous proof of concept examples at test tube and in vitro level, and some promising in vivo results, the field still faces several important challenges. For instance, the heat generated by the MNPs alone must be always carefully assessed in order to establish its role in the cellular death. In several of the examples discussed in this review it has been shown that sometimes MNPs alone are able to induce a high extent of cellular damage, while in others the cell death could not be unequivocally attributed to the released chemotherapeutics, as no sufficient data on the effect of the MNP heating alone were provided. Special attention must be also paid to the melting temperatures of the thermo-sensitive molecules used in the design of the nanocarriers for in vivo applications. On the one hand the premature release of the drug at physiological temperature must be avoided, while on the other hand the release should take place at clinically relevant 
A)

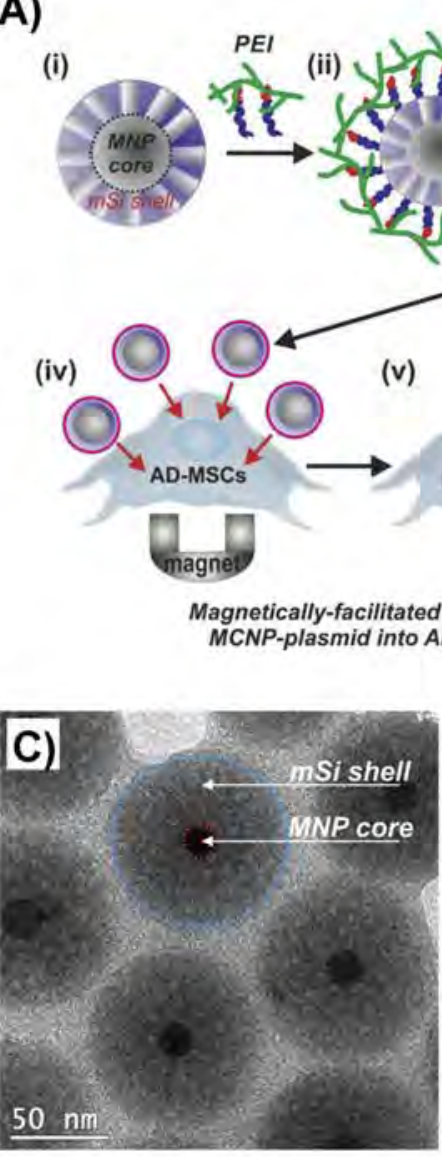

B) Heat-Inducible Plasmidy (ii

HSP70B' promoter

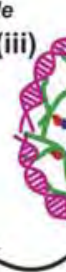

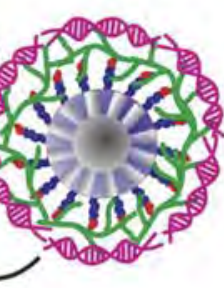

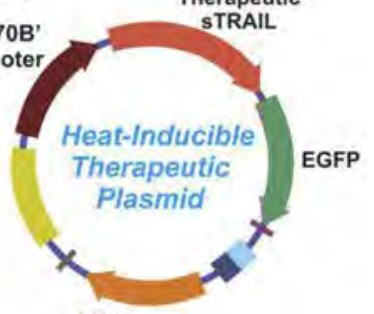

(vi)

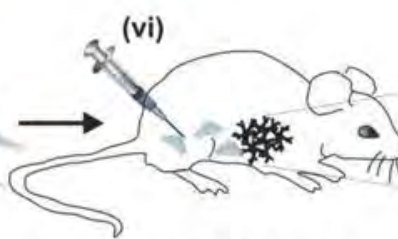

Engineered AD-MSCs migrate to tumors following IP injection

Exposure of the AD-MSCs to an AMF induces TRAIL secretion

D)

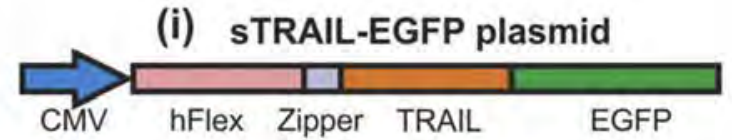

(ii) HSP-sTRAIL plasmid

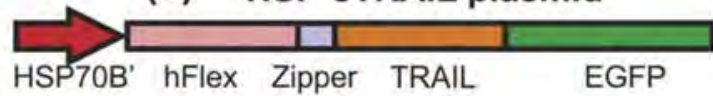

E)

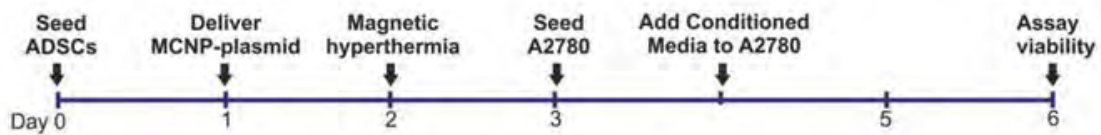

F)
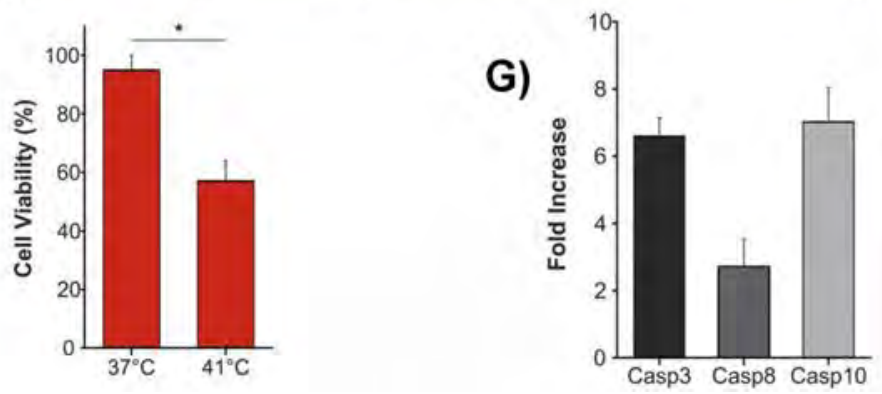

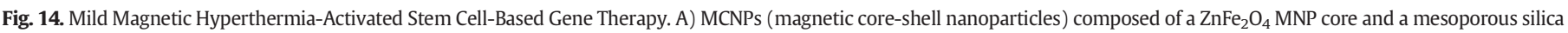

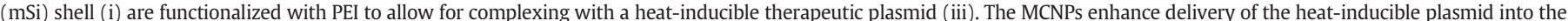

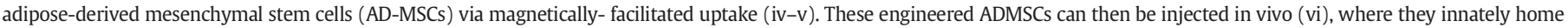

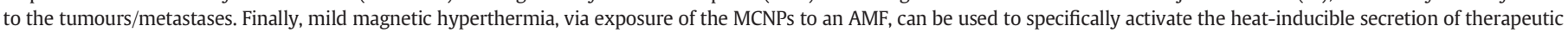

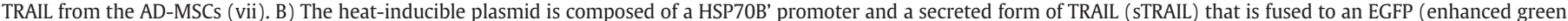

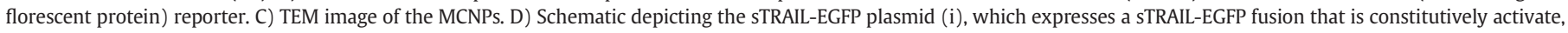

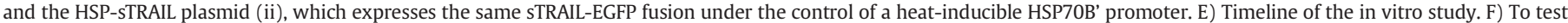

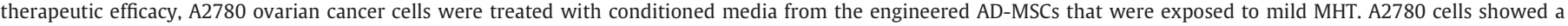

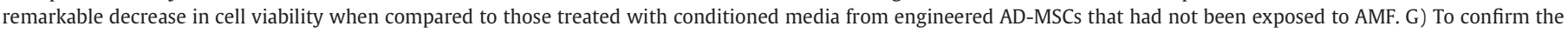

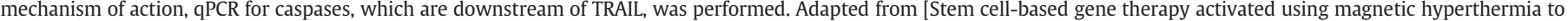
enhance the treatment of cancer] Licensed Content Publisher John Wiley and Sons. [113].

hyperthermia values $\left(41-44^{\circ} \mathrm{C}\right)$. Another major problem that renders the comparison of results obtained by different groups practically impossible is related to the parameters used for magnetic hyperthermia experiments (in terms of magnetic field amplitude and frequency, duration of application of the AMF, number of applications). Another problem is the low drug loading achieved in some cases, which may not be enough to achieve a successful treatment. Finally, given the complexity of cancer, it is also complicated to compare results from research works using different tumour cell lines.

As a final critical reflection, we believe that due to their rather large structural complexity the nanocarriers described in this review are at the moment in their very incipient stages of clinical development. However, we think that the field of thermo-responsive nanomaterials will continue to be a very prolific research area in the coming years and 
we are confident that by addressing all the afore mentioned challenges, the next generations of heat-responsive nanoplatforms could provide new solutions for cancer treatment.

\section{Acknowledgements}

L.G. and R.M.F. acknowledge financial support from the Ramón y Cajal subprogram (RYC-2014-15512 and RYC-2015-17640). E. M-A. acknowledges financial support from the Asociación Española contra el Cáncer (AECC), CSIC (Programa JAE-Intro) and ICMA (Programa de Iniciación a la Investigación). L.B. acknowledges financial support from Santander fellowships. M.M. acknowledges financial support from the Juan de la Cierva subprogram.

\section{Funding sources}

The present work was supported by grants from the Spanish MINECO (SAF2014-54763-C2-2-R and BIO2017-84246-C21-R), Fondo Social de la DGA (grupos DGA), COST Action TD1402 (Radiomag), and the European Commission through the M-ERA.NET COFUND project MagicCellGene (PCIN-2017-060).

\section{References}

[1] M. Colombo, S. Carregal-Romero, M.F. Casula, L. Gutierrez, M.P. Morales, I.B. Bohm, J.T. Heverhagen, D. Prosperi, W.J. Parak, Biological applications of magnetic nanoparticles, Chem. Soc. Rev. 41 (2012) 4306-4334, https://doi.org/10.1039/ C2CS15337H.

[2] D. Bobo, K.J. Robinson, J. Islam, K.J. Thurecht, S.R. Corrie, Nanoparticle-based medicines: a review of FDA-approved materials and clinical trials to date, Pharm. Res. 33 (2016) 2373-2387.

[3] R. Mejías, S. Pérez-Yagüe, L. Gutiérrez, L.I. Cabrera, R. Spada, P. Acedo, C.J. Serna, F.J Lázaro, Á. Villanueva, M. del P. Morales, D.F. Barber, Dimercaptosuccinic acidcoated magnetite nanoparticles for magnetically guided in vivo delivery of interferon gamma for cancer immunotherapy, Biomaterials 32 (2011) 2938-2952, https://doi.org/10.1016/J.BIOMATERIALS.2011.01.008.

[4] A. Jordan, R. Scholz, P. Wust, H. Fähling, Roland Felix, Magnetic fluid hyperthermia (MFH): Cancer treatment with AC magnetic field induced excitation of biocompatible superparamagnetic nanoparticles, J. Magn. Magn. Mater. 201 (1999) 413-419, https://doi.org/10.1016/S0304-8853(99)00088-8.

[5] S. Laurent, S. Dutz, U.O. Häfeli, M. Mahmoudi, Magnetic fluid hyperthermia: Focus on superparamagnetic iron oxide nanoparticles, Adv. Colloid Interf. Sci. 166 (2011) 8-23, https://doi.org/10.1016/J.CIS.2011.04.003.

[6] R. Hergt, S. Dutz, M. Robert, M. Zeisberger, Magnetic particle hyperthermia : nanoparticle magnetism and materials development for, cancer therapy 18 (2006) 2919-2934, https://doi.org/10.1088/0953-8984/18/38/S26.

[7] L. Néel, Influence des fluctuations thermiques a l'aimantation des particules ferromagn'etiques, Comptes Rendus de l'Académie des Sciences - Series IIB - Mechanics Volume: 228, 1949, (ISSN: 1620-7742 Online ISSN: 1873-751X).

[8] W.F. Brown Jr., Thermal fluctuations of a single-domain particle, Phys. Rev. 130 (5) (1963) 1677, https://doi.org/10.1103/PhysRev.130.1677.

[9] R. Hergt, S. Dutz, Magnetic particle hyperthermia-biophysical limitations of a visionary tumour therapy, J. Magn. Magn. Mater. 311 (2007) 187-192, https://doi. org/10.1016/J.JMMM.2006.10.1156.

[10] A. Attaluri, R. Ma, Y. Qiu, W. Li, L. Zhu, Nanoparticle distribution and temperature elevations in prostatic tumours in mice during magnetic nanoparticle hyperthermia, Int. J. Hyperth. 27 (2011) 491-502, https://doi.org/10.3109/02656736.2011. 584856.

[11] B. Hildebrandt, P. Wust, O. Ahlers, A. Dieing, G. Sreenivasa, T. Kerner, R. Felix, H. Riess, The cellular and molecular basis of hyperthermia, Crit. Rev. Oncol. / Hematol. 43 (2002) 33-56, https://doi.org/10.1016/S1040-8428(01)00179-2.

[12] M. Johannsen, U. Gneveckow, L. Eckelt, A. Feussner, N. Waldöfner, R. Scholz, S. Deger, P. Wust, S.A. Loening, A. Jordan, Clinical hyperthermia of prostate cancer using magnetic nanoparticles: Presentation of a new interstitial technique, Int. J. Hyperth. 21 (2005) 637-647, https://doi.org/10.1080/02656730500158360.

[13] D. Mertz, O. Sandre, S. Bégin-Colin, Drug releasing nanoplatforms activated by alternating magnetic fields, Biochim. Biophys. Acta - Gen. Subj. 1861 (2017) 1617-1641, https://doi.org/10.1016/j.bbagen.2017.02.025.

[14] T. Kang, F. Li, S. Baik, W. Shao, D. Ling, Biomaterials Surface design of magnetic nanoparticles for stimuli-responsive cancer imaging and therapy, Biomaterials 136 (2017) 98-114, https://doi.org/10.1016/j.biomaterials.2017.05.013.

[15] W. Guo, C. Yang, H. Lin, F. Qu, P(EO-co-LLA) functionalized Fe304@mSiO2 nanocomposites for thermo/pH responsive drug controlled release and hyperthermia, Dalton Trans. 43 (2014) 18056-18065, https://doi.org/10.1039/c4dt02441a.

[16] M. Santha Moorthy, S. Bharathiraja, P. Manivasagan, K.D. Lee, J. Oh, Crown ether triad modified core-shell magnetic mesoporous silica nanocarrier for $\mathrm{pH}$ responsive drug delivery and magnetic hyperthermia applications, New J. Chem. 41 (2017) 10935-10947, https://doi.org/10.1039/C7NJ02432K.
[17] S.R. Gayam, P. Venkatesan, Y.-M. Sung, S.-Y. Sung, S.-H. Hu, H.-Y. Hsu, S.-P. Wu, An $\mathrm{NAD}(\mathrm{P}) \mathrm{H}$ :quinone oxidoreductase 1 (NQO1) enzyme responsive nanocarrier based on mesoporous silica nanoparticles for tumor targeted drug delivery in vitro and in vivo, Nanoscale 8 (2016) 12307-12317, https://doi.org/10.1039/ C6NR03525F.

[18] Y.J. Cheng, G.F. Luo, J.Y. Zhu, X.D. Xu, X. Zeng, D.B. Cheng, Y.M. Li, Y. Wu, X.Z. Zhang, R.X. Zhuo, F. He, Enzyme-induced and tumor-targeted drug delivery system based on multifunctional mesoporous silica nanoparticles, ACS Appl. Mater. Interfaces 7 (2015) 9078-9087, https://doi.org/10.1021/acsami.5b00752.

[19] V.P. Torchilin, Multifunctional, stimuli-sensitive nanoparticulate systems for drug delivery, Nat. Publ. Gr. 13 (2014) 813-827, https://doi.org/10.1038/nrd4333.

[20] Y. Wang, D.S. Kohane, External triggering and triggered targeting strategies for drug delivery, Nat. Rev. Mater. 2 (2017), 17020. https://doi.org/10.1038/ natrevmats.2017.20.

[21] S. Mura, J. Nicolas, P. Couvreur, Stimuli-responsive nanocarriers for drug delivery, Nat. Publ. Gr. 12 (2013) 991-1003, https://doi.org/10.1038/nmat3776.

[22] ClinicalTrials.gov Search Results 09/05/2018, (2018) 2181075.

[23] Z.W. Tay, P. Chandrasekharan, A. Chiu-Lam, D.W. Hensley, R. Dhavalikar, X.Y. Zhou, E.Y. Yu, P.W. Goodwill, B. Zheng, C. Rinaldi, S.M. Conolly, Magnetic particle imaging-guided heating in vivo using gradient fields for arbitrary localization of magnetic hyperthermia therapy, 2018https://doi.org/10.1021/acsnano.8b00893.

[24] R.D. Issels, Hyperthermia adds to chemotherapy, Eur. J. Cancer 44 (2008) 2546-2554, https://doi.org/10.1016/j.ejca.2008.07.038.

[25] G.M. Hahn, Potential for Therapy of Drugs and Hyperthermia, Vol. 39, 1979 2264-2268.

[26] J. van der Zee, Heating the patient: a promising approach? Ann. Oncol. 13 (2002) 1173-1184, https://doi.org/10.1093/annonc/mdf280.

[27] T.-J. Li, C.-C. Huang, P.-W. Ruan, K.-Y. Chuang, K.-J. Huang, D.-B. Shieh, C.-S. Yeh, In vivo anti-cancer efficacy of magnetite nanocrystal - based system using locoregional hyperthermia combined with 5-fluorouracil chemotherapy, Biomaterials 34 (2013) 7873-7883, https://doi.org/10.1016/j.biomaterials.2013.07.012.

[28] J.-H. Lee, K.-J. Chen, S.-H. Noh, M.A. Garcia, H. Wang, W.-Y. Lin, H. Jeong, B.J. Kong, D.B. Stout, J. Cheon, H.-R. Tseng, On-demand Drug Release System for in Vivo Cancer Treatment through Self-Assembled magnetic Nanoparticles, Angew. Chem., Int. Ed. Engl. 52 (2013) 4384-4388, https://doi.org/10.1002/anie.201207721.

[29] J.T. Dias, M. Moros, P. Del Pino, S. Rivera, V. Grazú, J.M. de la Fuente, DNA as a molecular local thermal probe for the analysis of magnetic hyperthermia, Angew. Chem., Int. Ed. Engl. 52 (2013) 11526-11529, https://doi.org/10.1002/anie. 201305835.

[30] A.M. Derfus, G. Von Maltzahn, T.J. Harris, T. Duza, K.S. Vecchio, E. Ruoslahti, S.N. Bhatia, Remotely triggered release from magnetic nanoparticles, Adv. Mater. 19 (2007) 3932-3936, https://doi.org/10.1002/adma.200700091.

[31] N. Griffete, J. Fresnais, A. Espinosa, C. Wilhelm, A. Bée, C. Ménager, Design of magnetic molecularly imprinted polymer nanoparticles for controlled release of doxorubicin under an alternative magnetic field in athermal conditions, Nanoscale 7 (2015) 18891-18896, https://doi.org/10.1039/C5NR06133D.

[32] K. Ulbrich, K. Holá, V. Šubr, A. Bakandritsos, J. Tuček, R. Zbořil, Targeted Drug delivery with Polymers and magnetic Nanoparticles: Covalent and Noncovalent Approaches, Release Control, and Clinical Studies, Chem. Rev. 116 (2016) 5338-5431, https://doi.org/10.1021/acs.chemrev.5b00589.

[33] C.M. Niemeyer, Semisynthetic DNA-protein conjugates for biosensing and nanofabrication, Angew. Chem., Int. Ed. Engl. 49 (2010) 1200-1216, https://doi. org/10.1002/anie.200904930.

[34] M. Banchelli, S. Nappini, C. Montis, M. Bonini, P. Canton, D. Berti, P. Baglioni, Magnetic nanoparticle clusters as actuators of ssDNA release, Phys. Chem. Chem. Phys. 16 (2014), 10023. https://doi.org/10.1039/c3cp55470h.

[35] N. Guldris, J. Gallo, L. García-Hevia, J. Rivas, M. Bañobre-López, L.M. Salonen, Orthogonal Clickable Iron Oxide Nanoparticle Platform for Targeting, Imaging, and On-demand Release, Chem. Eur. J. (2018)https://doi.org/10.1002/chem. 201800389. doi:10.1002/chem.201800389.

[36] D. Yoo, H. Jeong, S.-H. Noh, J.-H. Lee, J. Cheon, Magnetically Triggered dual Functional Nanoparticles for Resistance-Free Apoptotic Hyperthermia, Angew. Chem. Int. Ed. Engl. 52 (2013) 13047-13051, https://doi.org/10.1002/anie.201306557.

[37] A. Riedinger, P. Guardia, A. Curcio, M.A. Garcia, R. Cingolani, L. Manna, T. Pellegrino, Subnanometer Local Temperature probing and Remotely Controlled Drug Release based on Azo-Functionalized Iron Oxide Nanoparticles, Nano Lett. 13 (2013) 2399-2406, https://doi.org/10.1021/nl400188q.

[38] M. Hammad, V. Nica, R. Hempelmann, On-command controlled drug release by diels-Alder reaction using Bi-magnetic core/shell nano-carriers, Colloids Surf. B. Biointerfaces. 150 (2017) 15-22, https://doi.org/10.1016/j.colsurfb.2016.11.005.

[39] J.M. Palomo, Diels-alder cycloaddition in protein chemistry, EurJOC. (2010) (2010) 6303-6314, https://doi.org/10.1002/ejoc.201000859.

[40] T.T.T. N'Guyen, H.T.T. Duong, J. Basuki, V. Montembault, S. Pascual, C. Guibert, J Fresnais, C. Boyer, M.R. Whittaker, T.P. Davis, L. Fontaine, Functional iron oxide magnetic nanoparticles with hyperthermia-induced drug release ability by using a combination of orthogonal click reactions, Angew. Chem., Int. Ed. Engl. 52 (2013) 14152-14156, https://doi.org/10.1002/anie.201306724.

[41] K. Seidel, A. Balakrishnan, C. Alexiou, C. Janko, R.M. Komoll, L.L. Wang, A Kirschning, M. Ott, Synthesis of Magnetic-Nanoparticle/Ansamitocin ConjugatesInductive heating leads to Decreased Cell Proliferation in Vitro and Attenuation of Tumour Growth in Vivo, Chem. Eur. J. 23 (2017) 12326-12337, https://doi. org/10.1002/chem.201701491.

[42] Y. Tabuchi, T. Kondo, Targeting heat shock transcription factor 1 for novel hyperthermia therapy, Int. J. Mol. Med. 32 (2013) 3-8, https://doi.org/10.3892/ijmm. 2013.1367. 
[43] C. Boyer, M.R. Whittaker, V. Bulmus, J. Liu, T.P. Davis, The design and utility of polymer-stabilized iron-oxide nanoparticles for nanomedicine applications, NPG Asia Mater. 2 (2010) 23-30, https://doi.org/10.1038/asiamat.2010.6.

[44] C.S.S.R. Kumar, F. Mohammad, Magnetic nanomaterials for hyperthermia-based therapy and controlled drug delivery, Adv. Drug Deliv. Rev. 63 (2011) 789-808, https://doi.org/10.1016/j.addr.2011.03.008.

[45] X. Ding, X. Shi, X. He, F. Yu, C. Xue, M. Liu, X. Cheng, J. Jia, H. Xin, X. Wang, Sol-gel system functionalized magnetic nanocubes as remote controlled drug carriers for cooperative tumor-targeted therapy, Mater. Lett. 175 (2016) 236-240, https:// doi.org/10.1016/j.matlet.2016.04.014.

[46] K. Hayashi, M. Nakamura, H. Miki, S. Ozaki, M. Abe, T. Matsumoto, W. Sakamoto, T. Yogo, K. Ishimura, Magnetically Responsive Smart Nanoparticles for Cancer Treatment with a Combination of magnetic Hyperthermia and Remote-Control Drug Release, Theranostics. 4 (2014) 834-844, https://doi.org/10.7150/thno.9199.

[47] O. Taratula, R.K. Dani, C. Schumann, H. Xu, A. Wang, H. Song, P. Dhagat, O. Taratula Multifunctional nanomedicine platform for concurrent delivery of chemotherapeutic drugs and mild hyperthermia to ovarian cancer cells, Int. J. Pharm. 458 (2013) 169-180, https://doi.org/10.1016/j.ijpharm.2013.09.032.

[48] H. Kakwere, M.P. Leal, M.E. Materia, A. Curcio, P. Guardia, D. Niculaes, R. Marotta, A Falqui, T. Pellegrino, Functionalization of strongly interacting magnetic nanocubes with (thermo)responsive coating and their application in hyperthermia and heattriggered drug delivery, ACS Appl. Mater. Interfaces 7 (2015) 10132-10145, https://doi.org/10.1021/am5088117.

[49] C.A. Cezar, S.M. Kennedy, M. Mehta, J.C. Weaver, L. Gu, H. Vandenburgh, D.J. Mooney, Biphasic Ferrogels for Triggered Drug and Cell Delivery, Adv. Healthc. Mater. 3 (2014) 1869-1876, https://doi.org/10.1002/adhm.201400095.

[50] E. Cazares-Cortes, A. Espinosa, J.M. Guigner, A. Michel, N. Griffete, C. Wilhelm, C. Ménager, Doxorubicin Intracellular Remote Release from Biocompatible Oligo(ethylene glycol) Methyl Ether Methacrylate-based magnetic Nanogels Triggered by magnetic Hyperthermia, ACS Appl. Mater. Interfaces 9 (2017) 25775-25788, https://doi.org/10.1021/acsami.7b06553.

[51] Y. Mi, X. Liu, J. Zhao, J. Ding, S.S. Feng, Multimodality treatment of cancer with herceptin conjugated, thermomagnetic iron oxides and docetaxel loaded nanoparticles of biodegradable polymers, Biomaterials 33 (2012) 7519-7529, https://doi. org/10.1016/j.biomaterials.2012.06.100.

[52] K. Fang, L. Song, Z. Gu, F. Yang, Y. Zhang, N. Gu, Magnetic field activated drug release system based on magnetic PLGA microspheres for chemo-thermal therapy, Colloids Surfaces B Biointerfaces. 136 (2015) 712-720, https://doi.org/10.1016/j. colsurfb.2015.10.014.

[53] Z.-Q. Zhang, S.-C. Song, Multiple hyperthermia-mediated release of TRAIL/SPION nanocomplex from thermosensitive polymeric hydrogels for combination cancer therapy, Biomaterials 132 (2017) 16-27, https://doi.org/10.1016/j.biomaterials 2017.03.049.

[54] A. Hervault, N.T.K. Thanh, Magnetic nanoparticle-based therapeutic agents for thermo-chemotherapy treatment of cancer, Nanoscale 6 (2014) 11553-11573, https://doi.org/10.1039/C4NR03482A.

[55] K. Oh, H. Baik, A. Lee, Y. Oh, Y. Youn, E. Lee, The Reversal of Drug-Resistance in Tumors using a Drug-carrying Nanoparticular System, Int. J. Mol. Sci. 10 (2009) 3776-3792, https://doi.org/10.3390/ijms10093776.

[56] G.M. Hahn, J. Braun, I. Har-Kedar, Thermochemotherapy: synergism between hyperthermia (42-43 degrees) and adriamycin (of bleomycin) in mammalian cell inactivation, Proc. Natl. Acad. Sci. 72 (1975) 937-940, https://doi.org/10.1073/pnas. 72.3.937.

[57] P. Wust, B. Hildebrandt, G. Sreenivasa, B. Rau, J. Gellermann, H. Riess, R. Felix, P. Schlag, Hyperthermia in combined treatment of cancer, Lancet Oncol. 3 (2002) 487-497, https://doi.org/10.1016/S1470-2045(02)00818-5.

[58] N.R. Datta, S.G. Ordóñez, U.S. Gaipl, M.M. Paulides, H. Crezee, J. Gellermann, D. Marder, E. Puric, S. Bodis, Local hyperthermia combined with radiotherapy and -/or chemotherapy: recent advances and promises for the future, Cancer Treat. Rev. 41 (2015) 742-753, https://doi.org/10.1016/j.ctrv.2015.05.009.

[59] R.W. Johnstone, A.J. Frew, M.J. Smyth, The TRAIL apoptotic pathway in cance onset, progression and therapy, Nat. Rev. Cancer 8 (2008) 782-798, https://doi. org/10.1038/nrc2465.

[60] J. Kydd, R. Jadia, P. Velpurisiva, A. Gad, S. Paliwal, P. Rai, Targeting strategies for the Combination Treatment of Cancer using Drug delivery Systems, Pharmaceutics. 9 (2017) 46, https://doi.org/10.3390/pharmaceutics9040046

[61] W. Xie, Q. Gao, Z. Guo, D. Wang, F. Gao, X. Wang, Y. Wei, L. Zhao, Injectable and selfhealing thermosensitive magnetic hydrogel for asynchronous control release of doxorubicin and docetaxel to treat triple-negative breast cancer, ACS Appl. Mater. Interfaces 9 (2017) 33660-33673, https://doi.org/10.1021/acsami.7b10699.

[62] G. Bozzuto, A. Molinari, Liposomes as nanomedical devices, Int. J. Nanomedicine 10 (2015) 975-999, https://doi.org/10.2147/IJN.S68861.

[63] D.C. M, J. M., Magnetoliposomes, Formation and structural characterization, Eur. Biophys. J. 15 (1988) 311-319.

[64] S. García-Jimeno, E. Escribano, J. Queralt, J. Estelrich, External magnetic fieldinduced selective biodistribution of magnetoliposomes in mice, Nanoscale Res. Lett. 7 (2012) 1-7, https://doi.org/10.1186/1556-276X-7-452.

[65] L. Zhang, S. Granick, How to stabilize phospholipid liposomes (using nanoparticles), Nano Lett. 6 (2006) 694-698, https://doi.org/10.1021/nl052455y.

[66] Y. Chen, Y. Chen, D. Xiao, A. Bose, R. Deng, G.D. Bothun, Low-dose chemotherapy of hepatocellular carcinoma through triggered-release from bilayer-decorated magnetoliposomes, Colloids Surfaces B Biointerfaces. 116 (2014) 452-458, https://doi.org/10.1016/j.colsurfb.2014.01.022.

[67] C.A. Monnier, D. Burnand, B. Rothen-Rutishauser, M. Lattuada, A. Petri-Fink, Magnetoliposomes: Opportunities and challenges, Eur. J. Nanomedicine. 6 (2014) 201-215, https://doi.org/10.1515/ejnm-2014-0042.
[68] B. Shirmardi Shaghasemi, M.M. Virk, E. Reimhult, Optimization of Magnetothermally Controlled Release Kinetics by Tuning of Magnetoliposome Composition and Structure, Sci. Rep. 7 (2017) 1-10, https://doi.org/10.1038/s41598-01706980-9.

[69] M. Pantusa, R. Bartucci, D. Marsh, L. Sportelli, Shifts in chain-melting transition temperature of liposomal membranes by polymer-grafted lipids, Biochim. Biophys. Acta Biomembr. 1614 (2003) 165-170, https://doi.org/10.1016/S0005-2736(03) 00171-8.

[70] V.V. Ginzburg, S. Balijepalli, Modeling the thermodynamics of the interaction of nanoparticles with cell membranes, Nano Lett. 7 (2007) 3716-3722, https://doi. org/10.1021/nl072053l.

[71] Y. Yu, S.M. Anthony, L. Zhang, S.C. Bae, S. Granick, Cationic nanoparticles stabilize zwitterionic liposomes better than anionic ones, J. Phys. Chem. C 111 (2007) 8233-8236, https://doi.org/10.1021/jp072680z.

72] M.R. Preiss, G.D. Bothun, Stimuli-responsive liposome-nanoparticle assemblies, Expert Opin. Drug Deliv. 8 (2011) 1025-1040, https://doi.org/10.1517/17425247. 2011.584868

[73] E. Amstad, J. Kohlbrecher, E. Müller, T. Schweizer, M. Textor, E. Reimhult, Triggered release from liposomes through magnetic actuation of iron oxide nanoparticle containing membranes, Nano Lett. 11 (2011) 1664-1670, https://doi.org/10.1021/ nl2001499.

[74] G.D. Bothun, M.R. Preiss, Bilayer heating in magnetite nanoparticle-liposome dispersions via fluorescence anisotropy, J. Colloid Interface Sci. 357 (2011) 70-74, https://doi.org/10.1016/j.jcis.2011.01.089.

[75] S. Nappini, F.B. Bombelli, M. Bonini, B. Nordèn, P. Baglioni, Magnetoliposomes for controlled drug release in the presence of low-frequency magnetic field, Soft Matter 6 (2010) 154-162, https://doi.org/10.1039/B915651H.

[76] Y. Chen, A. Bose, G.D. Bothun, Controlled Release from Bilayer-, ACS Nano, Vol. 4, 2010 3215-3221, https://doi.org/10.1021/nn100274v.

[77] G. Podaru, S. Ogden, A. Baxter, T. Shrestha, S. Ren, P. Thapa, R.K. Dani, H. Wang, M.T. Basel, P. Prakash, S.H. Bossmann, V. Chikan, Pulsed magnetic field induced fast drug release from magneto liposomes via ultrasound generation, J. Phys. Chem. B 118 (2014) 11715-11722, https://doi.org/10.1021/jp5022278.

[78] P. Pradhan, J. Giri, F. Rieken, C. Koch, O. Mykhaylyk, M. Döblinger, R. Banerjee, D. Bahadur, C. Plank, Targeted temperature sensitive magnetic liposomes for thermo-chemotherapy, J. Control. Release 142 (2010) 108-121, https://doi.org/ 10.1016/j.jconrel.2009.10.002.

[79] G.D. Bothun, A. Lelis, Y. Chen, K. Scully, L.E. Anderson, M.A. Stoner, Multicomponent folate-targeted magnetoliposomes: Design, characterization, and cellular uptake, Nanomedicine Nanotechnology, Biol. Med. 7 (2011) 797-805, https://doi.org/10. 1016/j.nano.2011.02.007.

[80] P. Kulshrestha, M. Gogoi, D. Bahadur, R. Banerjee, In vitro application of paclitaxel loaded magnetoliposomes for combined chemotherapy and hyperthermia, Colloids Surfaces B Biointerfaces. 96 (2012) 1-7, https://doi.org/10.1016/j.colsurfb. 2012.02.029.

81] A. Hardiansyah, L.-Y. Huang, M.-C. Yang, T.-Y. Liu, S.-C. Tsai, C.-Y. Yang, C.-Y. Kuo, T.Y. Chan, H.-M. Zou, W.-N. Lian, C.-H. Lin, Magnetic liposomes for colorectal cancer cells therapy by high-frequency magnetic field treatment, Nanoscale Res. Lett. 9 (2014) 497, https://doi.org/10.1186/1556-276X-9-497.

[82] A. Hardiansyah, M.C. Yang, T.Y. Liu, C.Y. Kuo, L.Y. Huang, T.Y. Chan, Hydrophobic Drug-Loaded PEGylated magnetic Liposomes for Drug-Controlled Release, Nanoscale Res. Lett. 12 (2017)https://doi.org/10.1186/s11671-017-2119-4.

[83] M. Yoshida, Y. Watanabe, M. Sato, T. Maehara, H. Aono, T. Naohara, H. Hirazawa, A. Horiuchl, S. Yukumi, K. Sato, H. Nakagawa, Y. Yamamoto, H. Sugishita, K. Kawachi, Feasibility of chemohyperthermia with docetaxel-embedded magnetoliposomes as minimally invasive local treatment for cancer, Int. J. Cancer 126 (2010) 1955-1965, https://doi.org/10.1002/ijc.24864.

[84] L. Wang, J. Zhang, Y. An, Z. Wang, J. Liu, Y. Li, D. Zhang, A study on the thermochemotherapy effect of nanosized As2O3/MZF thermosensitive magnetoliposomes on experimental hepatoma in vitro and in vivo, Nanotechnology 22 (2011)https://doi.org/10.1088/0957-4484/22/31/315102.

[85] M. Gogoi, M.K. Jaiswal, H.D. Sarma, D. Bahadur, R. Banerjee, Biocompatibility and therapeutic evaluation of magnetic liposomes designed for self-controlled cancer hyperthermia and chemotherapy, Integr. Biol. 9 (2017) 555-565, https://doi.org/ 10.1039/C6IB00234J.

[86] R. Di Corato, G. Béalle, J. Kolosnjaj-Tabi, A. Espinosa, O. Clément, A.K.A. Silva, C. Ménager, $C$. Wilhelm, Combining magnetic hyperthermia and photodynamic therapy for tumor ablation with photoresponsive magnetic liposomes, ACS Nano 9 (2015) 2904-2916, https://doi.org/10.1021/nn506949t.

[87] D. Press, The theranostic efficiency of tumor-specific, $\mathrm{pH}-$ paclitaxel and superparamagnetic iron oxide nanoparticles, 2018 1495-1504.

[88] Y. Guo, Y. Zhang, J. Ma, Q. Li, Y. Li, X. Zhou, D. Zhao, H. Song, Q. Chen, X. Zhu, Light/magnetic hyperthermia triggered drug released from multi-functional thermo-sensitive magnetoliposomes for precise cancer synergetic theranostics, J. Control. Release 272 (2018) 145-158, https://doi.org/10.1016/j.jconrel.2017. 04.028 .

[89] P.-J. Chen, S.-H. Hu, C.-S. Hsiao, Y.-Y. Chen, D.-M. Liu, S.-Y. Chen, Multifunctional magnetically removable nanogated lids of Fe304-capped mesoporous silica nanoparticles for intracellular controlled release and MR imaging, J. Mater. Chem. 21 (2011) 2535, https://doi.org/10.1039/c0jm02590a.

[90] P. Saint-Cricq, S. Deshayes, J.I. Zink, A.M. Kasko, Magnetic field activated drug delivery using thermodegradable azo-functionalised PEG-coated core-shell mesoporous silica nanoparticles, Nanoscale 7 (2015) 13168-13172, https://doi.org/10. 1039/C5NR03777H.

[91] C.R. Thomas, D.P. Ferris, J. Lee, E. Choi, M.H. Cho, E.S. Kim, J.F. Stoddart, J. Shin, J. Cheon, J.I. Zink, Noninvasive remote controlled release of drug molecules in vitro 
using magnetic actuation of mechanised nanoparticles, J. Am. Chem. Soc. 132 (2010) 10623-10625.

[92] B. Rühle, S. Datz, C. Argyo, T. Bein, J.I. Zink, A molecular nanocap activated by superparamagnetic heating for externally stimulated cargo release, Chem. Commun. 52 (2016) 1843-1846, https://doi.org/10.1039/C5CC08636A.

[93] A. Baeza, E. Guisasola, E. Ruiz-Hernández, M. Vallet-Regí, Magnetically Triggered Multidrug Release by Hybrid Mesoporous Silica Nanoparticles, Chem. Mater. 24 (2012) 517-524, https://doi.org/10.1021/cm203000u.

[94] Y. Zhu, C. Tao, DNA-capped $\mathrm{Fe}_{3} \mathrm{O}_{4} / \mathrm{SiO}_{2}$ magnetic mesoporous silica nanoparticles for potential controlled drug release and hyperthermia, RSC Adv. 5 (2015) 22365-22372, https://doi.org/10.1039/C5RA00701A.

[95] E. Ruiz-Hernández, A. Baeza, M. Vallet-Regí, Smart Drug delivery through DNA/ magnetic Nanoparticle Gates, ACS Nano 5 (2011) 1259-1266, https://doi.org/10. 1021/jp2059408.

[96] I.I. Slowing B.G. Trewyn, S. Giri, V.S.Y. Lin, Mesoporous silica nanoparticles for drug delivery and biosensing applications, Adv. Funct. Mater. 17 (2007) 1225-1236, https://doi.org/10.1002/adfm.200601191.

[97] A. Baeza, E. Guisasola, E. Ruiz-Hernández, M. Vallet-Regí, Magnetically triggered multidrug release by rybrid mesoporous silica nanoparticles, Chem. Mater. 24 (2012) 517-524, https://doi.org/10.1021/cm203000u.

[98] F. Benyettou, J.A. Ocadiz Flores, F. Ravaux, R. Rezgui, M. Jouiad, S.I. Nehme, R.K. Parsapur, J.C. Olsen, P. Selvam, A. Trabolsi, Mesoporous $\gamma$-Iron Oxide Nanoparticles for Magnetically Triggered Release of Doxorubicin and Hyperthermia Treatment, Chem. - A Eur.J. 22 (2016) 17020-17028, https://doi.org/10.1002/chem.201602956.

[99] Q. Zhang, J. Liu, K. Yuan, Z. Zhang, X. Zhang, X. Fang, A multi-controlled drug delivery system based on magnetic mesoporous Fe 304 nanopaticles and a phase change material for cancer thermo-chemotherapy, Nanotechnology 28 (2017), 405101. https://doi.org/10.1088/1361-6528/aa883f.

[100] H. Wang, O. Mu, R. Revia, K. Wang, X. Zhou, P.J. Pauzauskie, S. Zhou, M. Zhang, Chitosan-Gated Magnetic-Responsive Nanocarrier for Dual-Modal Optical Imaging, Switchable Drug Release, and Synergistic Therapy, Adv. Healthc. Mater. 6 (2017) 1-11, https://doi.org/10.1002/adhm.201601080.

[101] E. Guisasola, L. Asín, L. Beola, J.M. De La Fuente, A. Baeza, M. Vallet Regí, Beyond Traditional Hyperthermia. In vivo Cancer Treatment with Magnetic-Responsive Mesoporous Silica Nanocarriers, ACS Appl. Mater. Interfaces (2018)https://doi.org/10. 1021/acsami.8b02398 (acsami.8b02398).

[102] B. Fan, J.F. Trant, G. Hemery, O. Sandre, E.R. Gillies, Thermo-responsive selfimmolative nanoassemblies: direct and indirect triggering, Chem. Commun. 53 (2017) 12068-12071, https://doi.org/10.1039/C7CC06410A.

[103] L. Naldini, Gene therapy returns to Centre stage, Nature 526 (2015) 351, https:// doi.org/10.1038/nature15818.

[104] M.H. Amer, Gene therapy for cancer: present status and future perspective, Mol. Cell. Ther. 2 (2014) 27, https://doi.org/10.1186/2052-8426-2-27.

[105] C. Monzel, C. Vicario, J. Piehler, M. Coppey, M. Dahan, Magnetic Control of Cellular Processes using Biofunctional Nanoparticles, Chem. Sci. 8 (2017) 7330-7338, https://doi.org/10.1039/C7SC01462G.
[106] V. Ortner, C. Kaspar, C. Halter, L. Töllner, O. Mykhaylyk, J. Walzer, W.H. Günzburg J.A. Dangerfield, C. Hohenadl, T. Czerny, Magnetic field-controlled gene expression in encapsulated cells, J. Control. Release 158 (2012) 424-432, https://doi.org/10. 1016/j.jconrel.2011.12.006.

[107] M.E. de Sousa, A. Carrea, P. Mendoza Zélis, D. Muraca, O. Mykhaylyk, Y.E. Sosa, R.G Goya, F.H. Sánchez, R.A. Dewey, M.B. Fernández Van Raap, Stress-Induced Gene Expression Sensing Intracellular heating Triggered by magnetic Hyperthermia, J. Phys. Chem. C 120 (2016) 7339-7348, https://doi.org/10.1021/acs.jpcc.5b12330.

[108] A. Ito, M. Shinkai, H. Honda, T. Kobayashi, Heat-inducible TNF- $\alpha$ gene therapy combined with hyperthermia using magnetic nanoparticles as a novel tumor-targeted therapy, Cancer Gene Ther. 8 (2001) 649-654, https://doi.org/10.1038/sj.cgt 7700357.

[109] M. Moros, A. Ambrosone, G. Stepien, F. Fabozzi, V. Marchesano, A. Castaldi, A. Tino, J. de la Fuente, C. Tortiglione, Deciphering intracellular events triggered by mild magnetic hyperthermia in vitro and in vivo, Nanomedicine (Lond.) 10 (2015) 2167-2183, https://doi.org/10.2217/nnm.15.70.

[110] W. Walther, U. Stein, Heat-responsive gene expression for gene therapy, Adv. Drug Deliv. Rev. 61 (2009) 641-649, https://doi.org/10.1016/j.addr.2009.02.009.

[111] Q. Tang, D. Zhang, X. Cong, M. Wan, L. Jin, Using thermal energy produced by irradiation of $\mathrm{Mn}-\mathrm{Zn}$ ferrite magnetic nanoparticles (MZF-NPs) for heat-inducible gene expression, Biomaterials 29 (2008) 2673-2679, https://doi.org/10.1016/j. biomaterials.2008.01.038.

[112] M. Yamaguchi, A. Ito, A. Ono, Y. Kawabe, M. Kamihira, Heat-Inducible Gene Expression System by applying Alternating magnetic Field to magnetic Nanoparticles, ACS Synth. Biol. 3 (2014) 273-279, https://doi.org/10.1021/sb4000838.

[113] P.T. Yin, S. Shah, N.J. Pasquale, O.B. Garbuzenko, T. Minko, K.-B. Lee, Stem cell-based gene therapy activated using magnetic hyperthermia to enhance the treatment of cancer, Biomaterials 81 (2016) 46-57, https://doi.org/10.1016/j.biomaterials.2015 11.023.

[114] Y. Shi, X. Pang, J. Wang, G. Liu, NanoTRAIL-Oncology: a Strategic Approach in Cancer Research and Therapy, Adv. Healthc. Mater. (2018), 1800053. https://doi.org/ 10.1002/adhm.201800053.

[115] X. Wu, S. Wang, M. Li, A. Wang, Y. Zhou, P. Li, Y. Wang, Nanocarriers for TRAIL delivery: driving TRAIL back on track for cancer therapy, Nanoscale 9 (2017) 13879-13904, https://doi.org/10.1039/C7NR04959E.

[116] K.V. Nemani, R.C. Ennis, K.E. Griswold, B. Gimi, Magnetic nanoparticle hyperthermia induced cytosine deaminase expression in microencapsulated E. coli for enzyme-prodrug therapy, J. Biotechnol. 203 (2015) 32-40, https://doi.org/10. 1016/j.jbiotec.2015.03.008.

[117] P. Erbs, E. Regulier, J. Kintz, P. Leroy, Y. Poitevin, F. Exinger, R. Jund, M. Mehtali, In vivo cancer gene therapy by adenovirus-mediated transfer of a bifunctional yeast cytosine deaminase/uracil phosphoribosyltransferase fusion gene, Cancer Res. 60 (2000) 143813-143822. 